\title{
Lagrangian mechanics and variational integrators on two-spheres
}

\author{
Taeyoung Lee ${ }^{1, *, \dagger}$, Melvin Leok ${ }^{2}$ and N. Harris McClamroch ${ }^{3}$ \\ ${ }^{1}$ Department of Mechanical and Aerospace Engineering, Florida Institute of Technology, \\ Melbourne, FL 320901, U.S.A. \\ ${ }^{2}$ Department of Mathematics, Purdue University, West Lafayette, IN 47907, U.S.A. \\ ${ }^{3}$ Department of Aerospace Engineering, University of Michigan, Ann Arbor, MI 48109, U.S.A.
}

\begin{abstract}
SUMMARY
Euler-Lagrange equations and variational integrators are developed for Lagrangian mechanical systems evolving on a product of two-spheres. The geometric structure of a product of two-spheres is carefully considered in order to obtain global equations of motion. Both continuous equations of motion and variational integrators completely avoid the singularities and complexities introduced by local parameterizations or explicit constraints. We derive global expressions for the Euler-Lagrange equations on two-spheres, which are more compact than existing equations written in terms of angles. Since the variational integrators are derived from Hamilton's principle, they preserve the geometric features of the dynamics such as symplecticity, momentum maps, or total energy, as well as the structure of the configuration manifold. Computational properties of the variational integrators are illustrated for several mechanical systems. In addition, Lie group variational integrators can be used to integrate Lagrangian flows on more general homogeneous spaces. This is achieved by lifting the discrete Hamilton's principle on homogeneous spaces to a discrete variational principle on the Lie group that is constrained by a discrete connection. Copyright (C) 2009 John Wiley \& Sons, Ltd.
\end{abstract}

Received 24 June 2008; Revised 19 November 2008; Accepted 12 February 2009

KEY WORDS: Lagrangian mechanics; geometric integrator; variational integrator; two-sphere; homogeneous manifold

\section{INTRODUCTION}

The two-sphere $\mathbb{S}^{2}$ is the set of all points in the Euclidean space $\mathbb{R}^{3}$, which are a unit distance from the origin. It is a two-dimensional manifold that is locally diffeomorphic to $\mathbb{R}^{2}$. Many classical

\footnotetext{
*Correspondence to: Taeyoung Lee, Department of Mechanical and Aerospace Engineering, Florida Institute of Technology, Melbourne, FL 320901, U.S.A.

†E-mail: tylee@umich.edu

Contract/grant sponsor: National Science Foundation; contract/grant numbers: DMS-0714223, DMS-0726263, DMS0747659, ECS-0244977, CMS-0555797
}

Copyright (C) 2009 John Wiley \& Sons, Ltd. 
and interesting mechanical systems, such as a spherical pendulum, a double spherical pendulum, and magnetic models, evolve on the two-sphere or on a product of two-spheres. In this paper, we derive Euler-Lagrange equations on configuration spaces of the form $\left(\mathbb{S}^{2}\right)^{n}$, for a positive integer $n$. We also develop geometric numerical integrators referred to as discrete Euler-Lagrange equations or variational integrators on $\left(\mathbb{S}^{2}\right)^{n}$.

In most of the literature that treats dynamic systems on $\left(\mathbb{S}^{2}\right)^{n}$, either $2 n$ angles or $n$ explicit equality constraints enforcing unit length are used to describe the configuration of the system $[1,2]$. These descriptions involve complicated trigonometric expressions and introduce additional complexity in analysis and computations. In this paper, we focus on developing continuous equations of motion and discrete equations of motion directly on $\left(\mathbb{S}^{2}\right)^{n}$, without the need of local parameterizations, constraints, or reprojections. This provides a remarkably compact form of the equations of motion.

Geometric numerical integrators are numerical integration algorithms that preserve the geometric structure of the continuous dynamics, such as invariants, symplecticity, and the configuration manifold [3]. Conventional numerical integrators construct a discrete approximation to the flow using only information about the vector field, and ignore the physical laws and the geometric properties inherent in the differential equations [4]. Consequently, they do not preserve important characteristics of the dynamics of the continuous equations of motion. In contrast, variational integrators are constructed by discretizing Hamilton's principle, rather than discretizing the continuous Euler-Lagrange equation $[5,6]$. Since they are developed by using a discrete version of a physical principle, the resulting integrators have the desirable property that they are symplectic and momentum preserving, and they exhibit good energy behavior for exponentially long times.

Geometric numerical integration on $\mathbb{S}^{2}$ has been studied in [7-9]. The two-sphere is a homogeneous manifold; the special orthogonal group $\mathrm{SO}(3)$ acts transitively on $\mathbb{S}^{2}$ and the Lie group methods [10] can be adapted to generate numerical flows on $\mathbb{S}^{2}$. Our $\mathbb{S}^{2}$ variational integrators are based on $\mathrm{SO}(3)$ integrators that represent rotations as orthogonal matrices that are obtained from exponentiating skew matrices, as opposed to quaternion-based representations or local coordinate charts such as Euler angles. This allows us to avoid unit length constraints for the quaternion representation and coordinate singularities such as gimbal locking in the Euler angle representation. An in-depth discussion of the representations of the rotation group can be found in [11], and the advantages of the orthogonal matrix representation in numerical integration are described in $[12,13]$.

In this paper, we study Lagrangian mechanical systems on $\left(\mathbb{S}^{2}\right)^{n}$. Thus, it is desirable to preserve the geometric properties of the dynamics, such as momentum map, symplecticity, and total energy, in addition to the structure of the configuration manifold [12]. We combine the approaches of geometric integrators on homogeneous manifolds and variational integrators to obtain variational integrators on $\left(\mathbb{S}^{2}\right)^{n}$ that preserve the geometric properties of the dynamics as well as the homogeneous structure of the configuration manifold $\left(\mathbb{S}^{2}\right)^{n}$ concurrently.

The main contributions of this paper are as follows: (i) In the continuous time setting, the global Euler-Lagrange equations on $\left(\mathbb{S}^{2}\right)^{n}$ are developed in a compact form without local parameterization or constraints. This provides insight into the global dynamics on $\left(\mathbb{S}^{2}\right)^{n}$, which is desirable for theoretical studies. (ii) As a geometric numerical integrator, the discrete Euler-Lagrange equations on $\left(\mathbb{S}^{2}\right)^{n}$ are unique in the sense that they conserve both the geometric properties of the dynamics and the manifold structure of $\left(\mathbb{S}^{2}\right)^{n}$ simultaneously. The exact geometric properties of the discrete flow not only generate improved qualitative behavior, but they also provide accurate 
and reliable computational results in long-time simulation. (iii) More generally, the Lie group variational integrators can be applied to homogeneous spaces through a constrained lifted discrete variational principle. This allows the construction of homogeneous variational integrators for homogeneous spaces such as the Stiefel and Grassmannian manifolds, which are of particular interest in many scientific and engineering applications such as statistical signal processing [14] or computer vision [15].

A substantial class of nontrivial configuration spaces that arise in mechanical systems, including robotic arms, satellites, and underwater vehicles, can be identified with products of homogeneous spaces and the Lie groups. By extending the applicability of the Lie group variational integrators to the Lagrangian flows on homogeneous spaces, this paper provides a systematic framework for modeling and simulating systems with complicated internal shape degrees of freedom (DOF) that have not been extensively studied due to the limitations of existing techniques. As demonstrated in the numerous examples presented in this paper, the proposed approach yields compact expressions that clarifies the manner in which the interconnections between bodies enter into the resulting equations of motion.

This paper is organized as follows. Lagrangian mechanics on $\left(\mathbb{S}^{2}\right)^{n}$ is described in Section 2. Variational integrators on $\left(\mathbb{S}^{2}\right)^{n}$ are developed in Section 3. Computational properties are illustrated for several mechanical systems, namely a double spherical pendulum, an $n$-body problem on a sphere, an interconnected system of spherical pendula, pure bending of a geometrically-exact elastic rod, a spatial array of magnetic dipoles, and molecular dynamics that evolves on a sphere. The general theory of Lagrangian mechanics on homogeneous spaces in both continuous and discrete time is described in Section 4.

\section{LAGRANGIAN MECHANICS ON $\left(\mathbb{S}^{2}\right)^{n}$}

In this section, continuous equations of motion for a mechanical system defined on $\left(\mathbb{S}^{2}\right)^{n}$ are developed in the context of Lagrangian mechanics. It is common in the published literature that the equations of motion are developed by using either two angles or a unit length constraint to characterize $\mathbb{S}^{2}$. Any description with two angles has singularities, and any trajectory near a singularity experiences numerical ill-conditioning. The unit length constraint leads to additional complexity in the numerical computations. We develop global continuous equations of motion without resorting to local parameterizations or constraints. To achieve this, it is critical to understand the global characteristics of a mechanical system on $\left(\mathbb{S}^{2}\right)^{n}$. This section provides a good background for understanding the theory of discrete Lagrangian mechanics on $\left(\mathbb{S}^{2}\right)^{n}$ to be introduced in the next section.

The two-sphere is the set of points that have the unit length from the origin of $\mathbb{R}^{3}$, i.e. $\mathbb{S}^{2}=\left\{q \in \mathbb{R}^{3} \mid q \cdot q=1\right\}$. The tangent space $T_{q} \mathbb{S}^{2}$ for $q \in \mathbb{S}^{2}$ is a plane tangent to the two-sphere at the point $q$. Thus, a curve $q: \mathbb{R} \rightarrow \mathbb{S}^{2}$ and its time derivative satisfy $q \cdot \dot{q}=0$. The time-derivative of a curve can be written as

$$
\dot{q}=\omega \times q
$$

where the angular velocity $\omega \in \mathbb{R}^{3}$ is constrained to be orthogonal to $q$, i.e. $q \cdot \omega=0$. The time derivative of the angular velocity is also orthogonal to $q$, i.e. $q \cdot \dot{\omega}=0$. 


\subsection{Euler-Lagrange equations on $\left(\mathbb{S}^{2}\right)^{n}$}

We consider a mechanical system on the configuration manifold $\mathbb{S}^{2} \times \cdots \times \mathbb{S}^{2}=\left(\mathbb{S}^{2}\right)^{n}$. We assume that the Lagrangian $L: T\left(\mathbb{S}^{2}\right)^{n} \rightarrow \mathbb{R}$ is given by the difference between a quadratic kinetic energy and a configuration-dependent potential energy as follows:

$$
L\left(q_{1}, \ldots, q_{n}, \dot{q}_{1}, \ldots, \dot{q}_{n}\right)=\frac{1}{2} \sum_{i, j=1}^{n} M_{i j} \dot{q}_{i} \cdot \dot{q}_{j}-V\left(q_{1}, \ldots, q_{n}\right)
$$

where $\left(q_{i}, \dot{q}_{i}\right) \in T \mathbb{S}^{2}$ for $i \in\{1, \ldots, n\}$ and $M_{i j} \in \mathbb{R}$ is the $i, j$ th element of a symmetric positive definite inertia matrix $M \in \mathbb{R}^{n \times n}$ for $i, j \in\{1, \ldots, n\}$. The configuration-dependent potential is denoted by $V:\left(\mathbb{S}^{2}\right)^{n} \rightarrow \mathbb{R}$.

The action integral is defined as the time integral of the Lagrangia, and the variation of the action integral leads to continuous equations of motion by applying Hamilton's principle. These are standard procedures to derive the Euler-Lagrange equations. The expression for the infinitesimal variation of $q_{i} \in \mathbb{S}^{2}$ should be carefully developed, since the configuration manifold is not a linear vector space. As in (1), the infinitesimal variation of $q_{i}$ can be written as a vector cross product,

$$
\delta q_{i}=\xi_{i} \times q_{i}
$$

where $\xi_{i} \in \mathbb{R}^{3}$ is constrained to be orthogonal to $q_{i}$, i.e. $\xi_{i} \cdot q_{i}=0$. From this, the expression for the infinitesimal variation of $\dot{q}_{i}$ is given by

$$
\delta \dot{q}_{i}=\dot{\xi}_{i} \times q_{i}+\xi_{i} \times \dot{q}_{i}
$$

These expressions are the key elements to obtaining global equations of motion on $\left(\mathbb{S}^{2}\right)^{n}$.

The variation of the Lagrangian can be written as

$$
\delta L=\sum_{i, j=1}^{n} \delta \dot{q}_{i} \cdot M_{i j} \dot{q}_{j}-\sum_{i=1}^{n} \delta q_{i} \cdot \frac{\partial V}{\partial q_{i}}
$$

where the symmetric property $M_{i j}=M_{j i}$ is used. Substituting (3) and (4) into this and using the vector identity $(a \times b) \cdot c=a \cdot(b \times c)$ for any $a, b, c \in \mathbb{R}^{3}$, we obtain

$$
\delta L=\sum_{i, j=1}^{n} \dot{\xi}_{i} \cdot\left(q_{i} \times M_{i j} \dot{q}_{j}\right)+\xi_{i} \cdot\left(\dot{q}_{i} \times M_{i j} \dot{q}_{j}\right)-\sum_{i=1}^{n} \xi_{i} \cdot\left(q_{i} \times \frac{\partial V}{\partial q_{i}}\right)
$$

Let $\mathfrak{5}$ be the action integral defined as $\mathfrak{5}=\int_{0}^{T} L\left(q_{1}, \ldots, q_{n}, \dot{q}_{1}, \ldots, \dot{q}_{n}\right) \mathrm{d} t$. Using the above equation and integrating by parts, the variation of the action integral is given by

$$
\delta\left(\mathfrak{5}=\left.\sum_{i, j=1}^{n} \xi_{i} \cdot\left(q_{i} \times M_{i j} \dot{q}_{j}\right)\right|_{0} ^{T}-\sum_{i=1}^{n} \int_{0}^{T} \xi_{i} \cdot\left[\left(q_{i} \times \sum_{j=1}^{n} M_{i j} \ddot{q}_{j}\right)+q_{i} \times \frac{\partial V}{\partial q_{i}}\right]\right.
$$

From Hamilton's principle, $\delta \mathfrak{G}=0$ for any $\xi_{i}$ vanishing at $t=0, T$. Since $\xi_{i}$ is orthogonal to $q_{i}$, the continuous equations of motion satisfy

$$
\left(q_{i} \times \sum_{j=1}^{n} M_{i j} \ddot{q}_{j}\right)+q_{i} \times \frac{\partial V}{\partial q_{i}}=c_{i}(t) q_{i}
$$


for some scalar-valued functions $c_{i}(t)$ for $i \in\{1, \ldots, n\}$. Taking the cross product of (5) and $q_{i}$ yields

$$
q_{i} \times\left(q_{i} \times \sum_{j=1}^{n} M_{i j} \ddot{q}_{j}\right)+q_{i} \times\left(q_{i} \times \frac{\partial V}{\partial q_{i}}\right)=0
$$

From the vector identity $a \times(b \times c)=(a \cdot c) b-(a \cdot b) c$ for any $a, b, c \in \mathbb{R}^{3}$, we have

$$
\begin{aligned}
q_{i} \times\left(q_{i} \times \ddot{q}_{i}\right) & =\left(q_{i} \cdot \ddot{q}_{i}\right) q_{i}-\left(q_{i} \cdot q_{i}\right) \ddot{q}_{i} \\
& =-\left(\dot{q}_{i} \cdot \dot{q}_{i}\right) q_{i}-\ddot{q}_{i}
\end{aligned}
$$

where we use the properties $(\mathrm{d} / \mathrm{d} t)\left(q_{i} \cdot \dot{q}_{i}\right)=q_{i} \cdot \ddot{q}_{i}+\dot{q}_{i} \cdot \dot{q}_{i}=0$ and $q_{i} \cdot q_{i}=1$. Substituting these into (6), we obtain an expression for $\ddot{q}_{i}$, which is summarized as follows.

\section{Proposition 1}

Consider a mechanical system on $\left(\mathbb{S}^{2}\right)^{n}$ whose Lagrangian is expressed as (2). The continuous equations of motion are given by

$$
M_{i i} \ddot{q}_{i}=q_{i} \times\left(q_{i} \times \sum_{\substack{j=1 \\ j \neq i}}^{n} M_{i j} \ddot{q}_{j}\right)-\left(\dot{q}_{i} \cdot \dot{q}_{i}\right) M_{i i} q_{i}+q_{i} \times\left(q_{i} \times \frac{\partial V}{\partial q_{i}}\right)
$$

for $i \in\{1, \ldots, n\}$. Equivalently, this can be written in the matrix form as

$$
\left[\begin{array}{cccc}
M_{11} I_{3 \times 3} & -M_{12} \hat{q}_{1} \hat{q}_{1} & \ldots & -M_{1 n} \hat{q}_{1} \hat{q}_{1} \\
-M_{21} \hat{q}_{2} \hat{q}_{2} & M_{22} I_{3 \times 3} & \ldots & -M_{2 n} \hat{q}_{2} \hat{q}_{2} \\
\vdots & \vdots & & \vdots \\
-M_{n 1} \hat{q}_{n} \hat{q}_{n} & -M_{n 2} \hat{q}_{n} \hat{q}_{n} & \ldots & M_{n n} I_{3 \times 3}
\end{array}\right]\left[\begin{array}{c}
\ddot{q}_{1} \\
\ddot{q}_{2} \\
\vdots \\
\ddot{q}_{n}
\end{array}\right]=\left[\begin{array}{c}
-\left(\dot{q}_{1} \cdot \dot{q}_{1}\right) M_{11} q_{1}+\hat{q}_{1}^{2} \frac{\partial V}{\partial q_{1}} \\
-\left(\dot{q}_{2} \cdot \dot{q}_{2}\right) M_{22} q_{2}+\hat{q}_{2}^{2} \frac{\partial V}{\partial q_{2}} \\
\vdots \\
-\left(\dot{q}_{n} \cdot \dot{q}_{n}\right) M_{n n} q_{n}+\hat{q}_{n}^{2} \frac{\partial V}{\partial q_{n}}
\end{array}\right]
$$

where the hat map $\hat{\imath}: \mathbb{R}^{3} \rightarrow \mathbb{R}^{3 \times 3}$ is defined such that $\hat{a} b=a \times b$ for any $a, b \in \mathbb{R}^{3}$.

Since $\dot{q}_{i}=\omega_{i} \times q_{i}$ for the angular velocity $\omega_{i}$ satisfying $q_{i} \cdot \omega_{i}=0$, we have

$$
\ddot{q}_{i}=\dot{\omega}_{i} \times q_{i}+\omega_{i} \times\left(\omega_{i} \times q_{i}\right)=\dot{\omega}_{i} \times q_{i}-\left(\omega_{i} \cdot \omega_{i}\right) q_{i}
$$

Substituting this into (5) and using the fact that $q_{i} \cdot \dot{\omega}_{i}=0$, we obtain continuous equations of motion in terms of the angular velocity.

\section{Corollary 1}

The continuous equations of motion given by (7) can be written in terms of the angular velocity as

$$
\begin{aligned}
M_{i i} \dot{\omega}_{i} & =\sum_{\substack{j=1 \\
j \neq i}}^{n}\left(M_{i j} q_{i} \times\left(q_{j} \times \dot{\omega}_{j}\right)+M_{i j}\left(\omega_{j} \cdot \omega_{j}\right) q_{i} \times q_{j}\right)-q_{i} \times \frac{\partial V}{\partial q_{i}} \\
\dot{q}_{i} & =\omega_{i} \times q_{i}
\end{aligned}
$$


for $i \in\{1, \ldots, n\}$. Equivalently, this can be written in the matrix form as

$$
\begin{gathered}
{\left[\begin{array}{cccc}
M_{11} I_{3 \times 3} & -M_{12} \hat{q}_{1} \hat{q}_{2} & \ldots & -M_{1 n} \hat{q}_{1} \hat{q}_{n} \\
-M_{21} \hat{q}_{2} \hat{q}_{1} & M_{22} I_{3 \times 3} & \ldots & -M_{2 n} \hat{q}_{2} \hat{q}_{n} \\
\vdots & \vdots & & \vdots \\
-M_{n 1} \hat{q}_{n} \hat{q}_{1} & -M_{n 2} \hat{q}_{n} \hat{q}_{2} & \ldots & M_{n n} I_{3 \times 3}
\end{array}\right]\left[\begin{array}{c}
\dot{\omega}_{1} \\
\dot{\omega}_{2} \\
\vdots \\
\dot{\omega}_{n}
\end{array}\right]} \\
=\left[\begin{array}{c}
\sum_{j=2}^{n} M_{1 j}\left(\omega_{j} \cdot \omega_{j}\right) \hat{q}_{1} q_{j}-\hat{q}_{1} \frac{\partial V}{\partial q_{1}} \\
\sum_{j=1, j \neq 2}^{n} M_{2 j}\left(\omega_{j} \cdot \omega_{j}\right) \hat{q}_{2} q_{j}-\hat{q}_{2} \frac{\partial V}{\partial q_{2}} \\
\vdots \\
\sum_{j=1}^{n-1} M_{n j}\left(\omega_{j} \cdot \omega_{j}\right) \hat{q}_{n} q_{j}-\hat{q}_{n} \frac{\partial V}{\partial q_{n}}
\end{array}\right]
\end{gathered}
$$

Equations (7)-(11) are global continuous equations of motion for a mechanical system on $\left(\mathbb{S}^{2}\right)^{n}$. They avoid singularities completely and they preserve the structure of $T\left(\mathbb{S}^{2}\right)^{n}$ automatically, if an initial condition is chosen properly. These equations are useful for understanding global characteristics of the dynamics. In addition, these expressions are dramatically more compact than the equations of motion written in terms of any local parameterization.

We need to check that the $3 n \times 3 n$ matrices given by the first terms of (8) and (11) are nonsingular. This is a property of the mechanical system itself, rather than a consequence of the particular form of equations of motion. For example, when $n=2$, it can be shown that

$$
\begin{aligned}
\operatorname{det}\left[\begin{array}{cc}
M_{11} I_{3 \times 3} & -M_{12} \hat{q}_{1} \hat{q}_{1} \\
-M_{12} \hat{q}_{2} \hat{q}_{2} & M_{22} I_{3 \times 3}
\end{array}\right] & =\operatorname{det}\left[\begin{array}{cc}
M_{11} I_{3 \times 3} & -M_{12} \hat{q}_{1} \hat{q}_{2} \\
-M_{12} \hat{q}_{2} \hat{q}_{1} & M_{22} I_{3 \times 3}
\end{array}\right] \\
& =M_{11}^{2} M_{22}^{2}\left(M_{11} M_{22}-M_{12}^{2}\left(q_{1} \cdot q_{2}\right)^{2}\right)\left(M_{11} M_{22}-M_{12}^{2}\right)
\end{aligned}
$$

Since the inertia matrix is symmetric positive definite, $M_{11}, M_{22}>0, M_{11} M_{22}>M_{12}^{2}$, and from the Cauchy-Schwarz inequality, $\left(q_{1} \cdot q_{2}\right)^{2} \leqslant\left(q_{1} \cdot q_{1}\right)\left(q_{2} \cdot q_{2}\right)=1$. Thus, the above matrices are nonsingular. One may show a similar property for $n>2$. Throughout this paper, it is assumed that the $3 n \times 3 n$ matrices given at the first terms of (8) and (11) are nonsingular. Under this assumption, the Legendre transformation given in the next subsection is a diffeomorphism; the Lagrangian is hyperregular.

\subsection{Legendre transformation}

The Legendre transformation of the Lagrangian gives an equivalent Hamiltonian form of equations of motion in terms of conjugate momenta if the Lagrangian is hyperregular. Here, we find expressions for the conjugate momenta, which are used in the following section for the discrete equations of motion. For $q_{i} \in \mathbb{S}^{2}$, the corresponding conjugate momentum $p_{i}$ lies in the dual space 
$T_{q_{i}}^{*} \mathbb{S}^{2}$. We identify the tangent space $T_{q_{i}} \mathbb{S}^{2}$ and its dual space $T_{q_{i}}^{*} \mathbb{S}^{2}$ by using the usual dot product in $\mathbb{R}^{3}$. The Legendre transformation is given by

$$
p_{i} \cdot \delta q_{i}=\mathbf{D}_{\dot{q}_{i}} L\left(q_{1}, \ldots, q_{n}, \dot{q}_{1}, \ldots, \dot{q}_{n}\right) \cdot \delta q_{i}=\sum_{j=1}^{n} M_{i j} \dot{q}_{j} \cdot \delta q_{i}
$$

which is satisfied for any $\delta q_{i}$ perpendicular to $q_{i}$. Here $\mathbf{D}_{\dot{q}_{i}} L$ denotes the derivative of the Lagrangian with respect to $\dot{q}_{i}$. The momentum $p_{i}$ is an element of the dual space identified with the tangent space and the component parallel to $q_{i}$ has no effect since $\delta q_{i} \cdot q_{i}=0$. As such, the vector representing $p_{i}$ is perpendicular to $q_{i}$ and $p_{i}$ is equal to the projection of $\sum_{j=1}^{n} M_{i j} \dot{q}_{j}$ onto the orthogonal complement to $q_{i}$,

$$
\begin{aligned}
p_{i} & =\sum_{j=1}^{n}\left(M_{i j} \dot{q}_{j}-\left(q_{i} \cdot M_{i j} \dot{q}_{j}\right) q_{i}\right)=\sum_{j=1}^{n}\left(\left(q_{i} \cdot q_{i}\right) M_{i j} \dot{q}_{j}-\left(q_{i} \cdot M_{i j} \dot{q}_{j}\right) q_{i}\right) \\
& =M_{i i} \dot{q}_{i}-q_{i} \times\left(q_{i} \times \sum_{\substack{j=1 \\
j \neq i}}^{n} M_{i j} \dot{q}_{j}\right)
\end{aligned}
$$

The time derivative of $p_{i}$ is given by

$$
\dot{p}_{i}=M_{i i} \ddot{q}_{i}-\dot{q}_{i} \times\left(q_{i} \times \sum_{\substack{j=1 \\ j \neq i}}^{n} M_{i j} \dot{q}_{j}\right)-q_{i} \times\left(\dot{q}_{i} \times \sum_{\substack{j=1 \\ j \neq i}}^{n} M_{i j} \dot{q}_{j}\right)-q_{i} \times\left(q_{i} \times \sum_{\substack{j=1 \\ j \neq i}}^{n} M_{i j} \ddot{q}_{j}\right)
$$

Substituting (7), and using the vector identity $a \times(b \times c)=(a \cdot c) b-(a \cdot b) c$, we obtain the following Hamilton's equations.

\section{Corollary 2}

Consider a mechanical system on $\left(\mathbb{S}^{2}\right)^{n}$ whose Lagrangian is expressed as (2). Hamilton's equations are given by

$$
\begin{aligned}
& p_{i}=M_{i i} \dot{q}_{i}-q_{i} \times\left(q_{i} \times \sum_{\substack{j=1 \\
j \neq i}}^{n} M_{i j} \dot{q}_{j}\right) \\
& \dot{p}_{i}=-\sum_{j=1}^{n}\left(\dot{q}_{i} \cdot M_{i j} \dot{q}_{j}\right) q_{i}-\sum_{\substack{j=1 \\
j \neq i}}^{n}\left(q_{i} \cdot M_{i j} \dot{q}_{j}\right) \dot{q}_{i}+q_{i} \times\left(q_{i} \times \frac{\partial U}{\partial q_{i}}\right)
\end{aligned}
$$

for $i \in\{1, \ldots, n\}$. Equivalently, (13) can be written in a matrix form as

$$
\left[\begin{array}{c}
\dot{q}_{1} \\
\dot{q}_{2} \\
\vdots \\
\dot{q}_{n}
\end{array}\right]=\left[\begin{array}{cccc}
M_{11} I_{3 \times 3} & -M_{12} \hat{q}_{1} \hat{q}_{1} & \ldots & -M_{1 n} \hat{q}_{1} \hat{q}_{1} \\
-M_{21} \hat{q}_{2} \hat{q}_{2} & M_{22} I_{3 \times 3} & \ldots & -M_{2 n} \hat{q}_{2} \hat{q}_{2} \\
\vdots & \vdots & & \vdots \\
-M_{n 1} \hat{q}_{n} \hat{q}_{n} & -M_{n 2} \hat{q}_{n} \hat{q}_{n} & \ldots & M_{n n} I_{3 \times 3}
\end{array}\right]^{-1}\left[\begin{array}{c}
p_{1} \\
p_{2} \\
\vdots \\
p_{n}
\end{array}\right]
$$




\section{VARIATIONAL INTEGRATORS ON $\left(\mathbb{S}^{2}\right)^{n}$}

The dynamics of Lagrangian and Hamiltonian systems on $\left(\mathbb{S}^{2}\right)^{n}$ have unique geometric properties: the Hamiltonian flow is symplectic, the total energy is conserved in the absence of nonconservative forces, and the momentum map associated with a symmetry of the system is preserved. The configuration space is a homogeneous manifold. These geometric features determine the qualitative dynamics of the system and serve as a basis for theoretical study.

Conventional numerical integrators construct a discrete approximation of the flow using only information about the vector field. Other than the direction specified by the vector field, they completely ignore the physical laws and the geometric properties inherent in the differential equations [4]. For example, if we integrate (11) by using an explicit Runge-Kutta method, the unit length of the vector $q_{i}$ and the total energy are not preserved numerically; we will see this later in this paper.

Numerical integration methods that preserve the symplecticity of a Hamiltonian system have been studied [16]. Coefficients of a Runge-Kutta method can be carefully chosen to satisfy a symplecticity criterion and order conditions to obtain a symplectic Runge-Kutta method. However, it can be difficult to construct such integrators, and it is not guaranteed that other invariants of the system, such as the momentum map, are preserved. Alternatively, variational integrators are constructed by discretizing Hamilton's principle, rather than by discretizing the continuous EulerLagrange equation $[5,6]$. The key feature of variational integrators is that they are derived by a discrete version of a physical principle, so that the resulting integrators satisfy the physical properties automatically in a discrete sense; they are symplectic and momentum preserving, and they exhibit good energy behavior for exponentially long times. The Lie group methods are numerical integrators that preserve the Lie group structure of the configuration space [10]. Recently, these two approaches have been unified to obtain the Lie group variational integrators that preserve the geometric properties of the dynamics as well as the Lie group structure of the configuration manifold [13].

The two-sphere is a homogeneous manifold. It does not have a Lie group structure by itself, but instead, the special orthogonal group, $\mathrm{SO}(3)=\left\{F \in \mathbb{R}^{3 \times 3} \mid F^{\mathrm{T}} F=I_{3 \times 3}\right.$, det $\left.F=1\right\}$, acts on $\mathbb{S}^{2}$ in a transitive way; for any $q_{1}, q_{2} \in \mathbb{S}^{2}$, there exists $F \in \mathrm{SO}(3)$ such that $q_{2}=F q_{1}$. If a group acts transitively on a manifold, a curve on the manifold can be represented as the action of a curve in the Lie group on an initial point on the manifold. As such, Lie group methods can be applied to obtain numerical integration schemes for homogeneous manifolds [7-9]. However, it is not guaranteed that these methods preserve the geometric properties of the dynamics. In this paper, we focus on a Lagrangian mechanical system evolving on the homogeneous manifold, $\left(\mathbb{S}^{2}\right)^{n}$, by extending the method of the Lie group variational integrators $[12,13]$. The resulting integrator preserves the dynamic characteristics and the homogeneous manifold structure concurrently.

\subsection{Discrete Euler-Lagrange equations on $\left(\mathbb{S}^{2}\right)^{n}$}

The procedure to derive discrete Euler-Lagrange equations follows the development of the continuous time case; the tangent bundle is replaced by a cartesian product of the configuration manifold; a discrete Lagrangian is chosen to approximate the integral of the Lagrangian over a discrete time step and the variation of the corresponding discrete action sum provides discrete Euler-Lagrange equations, referred to as a variational integrator. The discrete version of the Legendre transformation yields the discrete equations in the Hamiltonian form. 
Let the number of timesteps be $N$, with constant timesteps $h>0$. A variable with subscript $k$ denotes the value of variable at $t=k h$. Define a discrete Lagrangian $L_{\mathrm{d}}:\left(\mathbb{S}^{2}\right)^{n} \times\left(\mathbb{S}^{2}\right)^{n} \rightarrow \mathbb{R}$ such that it approximates the integral of the Lagrangian given by (2) over a discrete time step

$$
L_{\mathrm{d}}\left(q_{1_{k}}, \ldots, q_{n_{k}}, q_{1_{k+1}}, \ldots, q_{n_{k+1}}\right)=\frac{1}{2 h} \sum_{i, j=1}^{n} M_{i j}\left(q_{i_{k+1}}-q_{i_{k}}\right) \cdot\left(q_{j_{k+1}}-q_{j_{k}}\right)-\frac{h}{2} V_{k}-\frac{h}{2} V_{k+1}
$$

where $V_{k}$ denotes the value of the potential at the $k$ th step, i.e. $V_{k}=V\left(q_{1_{k}}, \ldots, q_{n_{k}}\right)$. As given in (3), the infinitesimal variation of $q_{i_{k}}$ is written as

$$
\delta q_{i_{k}}=\xi_{i_{k}} \times q_{i_{k}}
$$

where $\xi_{i_{k}} \in \mathbb{R}^{3}$ is constrained to be orthogonal to $q_{i_{k}}$, i.e. $\xi_{i_{k}} \cdot q_{i_{k}}=0$. The variation of the discrete Lagrangian can be written as

$$
\delta L_{\mathrm{d}_{k}}=\frac{1}{h} \sum_{i, j=1}^{n}\left(\delta q_{i_{k+1}}-\delta q_{i_{k}}\right) \cdot M_{i j}\left(q_{j_{k+1}}-q_{j_{k}}\right)-\frac{h}{2} \sum_{i=1}^{n}\left(\delta q_{i_{k}} \cdot \frac{\partial V_{k}}{\partial q_{i_{k}}}+\delta q_{i_{k+1}} \cdot \frac{\partial V_{k+1}}{\partial q_{i_{k+1}}}\right)
$$

Substituting (17) into (18), and using the vector identity $(a \times b) \cdot c=a \cdot(b \times c)$ for any $a, b, c \in \mathbb{R}^{3}$, we obtain

$$
\begin{aligned}
\delta L_{\mathrm{d}_{k}}= & \frac{1}{h} \sum_{i, j=1}^{n}\left(\xi_{i_{k+1}} \cdot\left(q_{i_{k+1}} \times M_{i j}\left(q_{j_{k+1}}-q_{j_{k}}\right)\right)-\xi_{i_{k}} \cdot\left(q_{i_{k}} \times M_{i j}\left(q_{j_{k+1}}-q_{j_{k}}\right)\right)\right) \\
& -\frac{h}{2} \sum_{i=1}^{n}\left(\xi_{i_{k}} \cdot\left(q_{i_{k}} \times \frac{\partial V_{k}}{\partial q_{i_{k}}}\right)+\xi_{i_{k+1}} \cdot\left(q_{i_{k+1}} \times \frac{\partial V_{k+1}}{\partial q_{i_{k+1}}}\right)\right)
\end{aligned}
$$

Let $\mathfrak{6}_{\mathrm{d}}$ be the discrete action sum defined as $\boldsymbol{6}_{\mathrm{d}}=\sum_{k=0}^{N-1} L_{\mathrm{d}_{k}}$, which approximates the action integral as the discrete Lagrangian approximates a piece of the action integral over a discrete time step. The variation of the action sum is obtained by using (19). Using the fact that $\xi_{i_{k}}$ vanish at $k=0$ and $k=N$, we can reindex the summation, which is the discrete analog of integration by parts, to yield

$$
\delta \mathfrak{G}_{\mathrm{d}}=\sum_{k=1}^{N-1} \sum_{i=1}^{n} \xi_{i_{k}} \cdot\left[\frac{1}{h}\left(q_{i_{k}} \times \sum_{j=1}^{n} M_{i j}\left(-q_{j_{k+1}}+2 q_{j_{k}}-q_{j_{k-1}}\right)\right)-h q_{i_{k}} \times \frac{\partial V_{k}}{\partial q_{i_{k}}}\right]
$$

From discrete Hamilton's principle, $\delta \mathfrak{G}_{\mathrm{d}}=0$ for any $\xi_{i_{k}}$ perpendicular to $q_{i_{k}}$. Using the same argument given in (5), the discrete equations of motion are given by

$$
\frac{1}{h}\left(q_{i_{k}} \times \sum_{j=1}^{n} M_{i j}\left(-q_{j_{k+1}}+2 q_{j_{k}}-q_{j_{k-1}}\right)\right)-h q_{i_{k}} \times \frac{\partial V_{k}}{\partial q_{i_{k}}}=0
$$

for $i \in\{1, \ldots, n\}$. In addition, we require that the unit length of the vector $q_{i_{k}}$ is preserved. This is achieved by viewing $\mathbb{S}^{2}$ as a homogeneous manifold. Since the special orthogonal group $\mathrm{SO}(3)$ acts on $\mathbb{S}^{2}$ transitively, we can define a discrete update map for $q_{i_{k}}$ as

$$
q_{i_{k+1}}=F_{i_{k}} q_{i_{k}}
$$


for $F_{i_{k}} \in \mathrm{SO}(3)$. Then, the unit length of the vector $q_{i}$ is preserved through the discrete equations of motion, since $q_{i_{k+1}} \cdot q_{i_{k+1}}=q_{i_{k}}^{\mathrm{T}} F_{i_{k}}^{\mathrm{T}} F_{i_{k}} q_{i_{k}}=1$. These results are summarized as follows.

Proposition 2

Consider a mechanical system on $\left(\mathbb{S}^{2}\right)^{n}$ whose Lagrangian is expressed as (2). The discrete equations of motion are given by

$$
\begin{aligned}
M_{i i} q_{i_{k}} \times F_{i_{k}} q_{i_{k}}+q_{i_{k}} \times \sum_{\substack{j=1 \\
j \neq i}}^{n} M_{i j}\left(F_{j_{k}}-I_{3 \times 3}\right) q_{j_{k}} & =q_{i_{k}} \times \sum_{j=1}^{n} M_{i j}\left(q_{j_{k}}-q_{j_{k-1}}\right)-h^{2} q_{i_{k}} \times \frac{\partial V_{k}}{\partial q_{i_{k}}} \\
q_{i_{k+1}} & =F_{i_{k}} q_{i_{k}}
\end{aligned}
$$

for $i \in\{1, \ldots, n\}$. For given $\left(q_{i_{k-1}}, q_{i_{k}}\right)$, we solve (21) to obtain $F_{i_{k}} \in \mathrm{SO}(3)$. Then, $q_{i_{k+1}}$ is computed by (22). This yields a discrete flow map $\left(q_{i_{k-1}}, q_{i_{k}}\right) \mapsto\left(q_{i_{k}}, q_{i_{k+1}}\right)$ and this process is repeated.

\section{Remark 1}

It should be noted that the equations given in Proposition 2 do not uniquely define the rotation matrices $F_{i_{k}}$, which are used to generate the updates on $\left(\mathbb{S}^{2}\right)^{n}$. This is since the Lie group action of $S O(3)$ on $\mathbb{S}^{2}$ has a nontrivial isotropy subgroup, corresponding to the fact that rotating a vector $q_{i}$ about the $q_{i}$ axis leaves the vector invariant. In order to make the equations well-defined, we need to choose a connection on $\operatorname{TSO}(3)$ that is complementary to the local isotropy direction, and requires that the rotation matrix used to update the solution is in the horizontal distribution associated with this connection. In Section 3.3, the connection is chosen by requiring that $F_{i}=\operatorname{Cay}\left(f_{i}\right)$, where $f_{i}$ is constrained to satisfy $f_{i} \cdot q_{i}=0$.

More generally, an appropriate connection can be constructed as follows: (i) the continuous horizontal distribution can be obtained by requiring that the element of the algebra $\xi \in \mathfrak{g}$ is orthogonal, with respect to a given inner product $\langle\cdot, \cdot\rangle$, to generators $h_{j}$ of the isotropy subalgebra $\mathfrak{h}$, i.e., $\left\langle\xi, h_{j}\right\rangle=0$, (ii) the continuous connection yields a discrete connection by choosing a retraction [17], $\varphi_{g}: T_{g} G \rightarrow G$ and requiring that the update element $F_{k} \in G$, where $g_{k+1}=F_{k} g_{k}$, satisfies $\left\langle\varphi_{g_{k}}^{-1}\left(F_{k}\right), h_{j}\right\rangle=0$. This construction is discussed in greater detail in Section 4.2.

\subsection{Discrete Legendre transformation}

We find discrete equations of motion in terms of the angular velocity. The discrete Legendre transformation is given as follows [6]:

$$
p_{i_{k}} \cdot \delta q_{i_{k}}=-\mathbf{D}_{q_{i_{k}}} L_{\mathrm{d}_{k}} \cdot \delta q_{i_{k}}=\left[\frac{1}{h} \sum_{j=1}^{n} M_{i j}\left(q_{j_{k+1}}-q_{j_{k}}\right)+\frac{h}{2} \frac{\partial V_{k}}{\partial q_{i_{k}}}\right] \cdot \delta q_{i_{k}}
$$

which can be directly obtained from (18). This is satisfied for any $\delta q_{i_{k}}$ perpendicular to $q_{i_{k}}$. Using the same argument used to derive (12), the conjugate momenta $p_{i_{k}}$ is the projection of the expression in brackets onto the orthogonal complement of $q_{i_{k}}$. Thus, we obtain

$$
p_{i_{k}}=-\frac{1}{h} q_{i_{k}} \times\left(q_{i_{k}} \times \sum_{j=1}^{n} M_{i j}\left(q_{j_{k+1}}-q_{j_{k}}\right)\right)-\frac{h}{2} q_{i_{k}} \times\left(q_{i_{k}} \times \frac{\partial V_{k}}{\partial q_{i_{k}}}\right)
$$


Similarly, we obtain

$$
p_{i_{k+1}} \cdot \delta q_{i_{k+1}}=\mathbf{D}_{q_{i_{k+1}}} L_{\mathrm{d}_{k}} \cdot \delta q_{i_{k+1}}=\left[\frac{1}{h} \sum_{j=1}^{n} M_{i j}\left(q_{j_{k+1}}-q_{j_{k}}\right)-\frac{h}{2} \frac{\partial V_{k+1}}{\partial q_{i_{k+1}}}\right] \cdot \delta q_{i_{k+1}}
$$

Since $p_{i_{k+1}}$ is perpendicular to $q_{i_{k+1}}$, it is given by

$$
p_{i_{k+1}}=-\frac{1}{h} q_{i_{k+1}} \times\left(q_{i_{k+1}} \times \sum_{j=1}^{n} M_{i j}\left(q_{j_{k+1}}-q_{j_{k}}\right)\right)+\frac{h}{2} q_{i_{k+1}} \times\left(q_{i_{k+1}} \times \frac{\partial V_{k+1}}{\partial q_{i_{k+1}}}\right)
$$

This yields the discrete-time Hamilton's equations as follows.

Corollary 3

Consider a mechanical system on $\left(\mathbb{S}^{2}\right)^{n}$ whose Lagrangian is expressed as (2). The discrete-time Hamilton's equations are given by

$$
\begin{aligned}
p_{i_{k}} & =-\frac{1}{h} q_{i_{k}} \times\left(q_{i_{k}} \times \sum_{j=1}^{n} M_{i j}\left(F_{j_{k}}-I_{3 \times 3}\right) q_{j_{k}}\right)-\frac{h}{2} q_{i_{k}} \times\left(q_{i_{k}} \times \frac{\partial V_{k}}{\partial q_{i_{k}}}\right) \\
q_{i_{k+1}} & =F_{i_{k}} q_{i_{k}} \\
p_{i_{k+1}} & =-\frac{1}{h} q_{i_{k+1}} \times\left(q_{i_{k+1}} \times \sum_{j=1}^{n} M_{i j}\left(q_{j_{k+1}}-q_{j_{k}}\right)\right)+\frac{h}{2} q_{i_{k+1}} \times\left(q_{i_{k+1}} \times \frac{\partial V_{k+1}}{\partial q_{i_{k+1}}}\right)
\end{aligned}
$$

for $i \in\{1, \ldots, n\}$. For given $\left(q_{i_{k}}, p_{i_{k}}\right)$, we solve (24) to obtain $F_{i_{k}} \in \operatorname{SO}(3)$. Then, $q_{i_{k+1}}$ and $p_{i_{k+1}}$ are computed by (25) and (26), respectively. This yields a discrete-time flow map $\left(q_{i_{k}}, p_{i_{k}}\right) \mapsto$ $\left(q_{i_{k+1}}, p_{i_{k+1}}\right)$ and this process is repeated.

This provides a discrete-time flow map in terms of the conjugate momenta. Now, we find a discrete-time flow map written in terms of the angular velocity. Comparing (24) with (12), substituting $\dot{q}_{i_{k}}=\omega_{i_{k}} \times q_{i_{k}}$ and rearranging, we obtain

$$
q_{j_{k}} \times\left[M_{i i} \omega_{i_{k}}+\left(q_{i_{k}} \times \sum_{\substack{j=1 \\ j \neq i}}^{n} M_{i j}\left(\omega_{j_{k}} \times q_{j_{k}}\right)\right)-\frac{1}{h}\left(q_{i_{k}} \times \sum_{j=1}^{n} M_{i j}\left(q_{j_{k+1}}-q_{j_{k}}\right)\right)-\frac{h}{2} q_{i_{k}} \times \frac{\partial V_{k}}{\partial q_{i_{k}}}\right]=0
$$

Since the expression in the brackets is orthogonal to $q_{i_{k}}$, the left-hand side is equal to zero if and only if the expression in the brackets is zero. Thus,

$$
M_{i i} \omega_{i_{k}}+\left(q_{i_{k}} \times \sum_{\substack{j=1 \\ j \neq i}}^{n} M_{i j}\left(\omega_{j_{k}} \times q_{j_{k}}\right)\right)=\frac{1}{h}\left(q_{i_{k}} \times \sum_{j=1}^{n} M_{i j}\left(q_{j_{k+1}}-q_{j_{k}}\right)\right)+\frac{h}{2} q_{i_{k}} \times \frac{\partial V_{k}}{\partial q_{i_{k}}}
$$

This provides a relationship between $\left(q_{i_{k}}, \omega_{i_{k}}\right)$ and $\left(q_{i_{k}}, q_{i_{k+1}}\right)$. Comparing this with (20), we obtain

$$
M_{i i} \omega_{i_{k}}+\left(q_{i_{k}} \times \sum_{\substack{j=1 \\ j \neq i}}^{n} M_{i j}\left(\omega_{j_{k}} \times q_{j_{k}}\right)\right)=\frac{1}{h}\left(q_{i_{k}} \times \sum_{j=1}^{n} M_{i j}\left(q_{j_{k}}-q_{j_{k-1}}\right)\right)-\frac{h}{2} q_{i_{k}} \times \frac{\partial V_{k}}{\partial q_{i_{k}}}
$$


which provides a relationship between $\left(q_{i_{k}}, \omega_{i_{k}}\right)$ and $\left(q_{i_{k-1}}, q_{i_{k}}\right)$. Equations (27) and (28) give a discrete flow map in terms of the angular velocity; for a given $\left(q_{i_{k}}, \omega_{i_{k}}\right)$, we find $\left(q_{i_{k}}, q_{i_{k+1}}\right)$ by using (27). Substituting this into (28) expressed at the $(k+1)$ th step, we obtain $\left(q_{i_{k+1}}, \omega_{i_{k+1}}\right)$. This procedure is summarized as follows.

\section{Corollary 4}

The discrete equations of motion given by (21) and (22) can be written in terms of the angular velocity as

$$
\begin{aligned}
M_{i i} q_{i_{k}} \times F_{i_{k}} q_{i_{k}}+q_{i_{k}} \times \sum_{\substack{j=1 \\
j \neq i}}^{n} M_{i j}\left(F_{j_{k}}-I_{3 \times 3}\right) q_{j_{k}}= & M_{i i} h \omega_{i_{k}}-\left(q_{i_{k}} \times \sum_{\substack{j=1 \\
j \neq i}}^{n} M_{i j}\left(q_{j_{k}} \times h \omega_{j_{k}}\right)\right) \\
& -\frac{h^{2}}{2} q_{i_{k}} \times \frac{\partial V_{k}}{\partial q_{i_{k}}} \\
q_{i_{k+1}}= & F_{i_{k}} q_{i_{k}} \\
M_{i i} \omega_{i_{k+1}}-\left(q_{i_{k+1}} \times \sum_{\substack{j=1 \\
j \neq i}}^{n} M_{i j}\left(q_{j_{k+1}} \times \omega_{j_{k+1}}\right)\right)= & \frac{1}{h}\left(q_{i_{k+1}} \times \sum_{j=1}^{n} M_{i j}\left(q_{j_{k+1}}-q_{j_{k}}\right)\right) \\
& -\frac{h}{2} q_{i_{k+1}} \times \frac{\partial V_{k+1}}{\partial q_{i_{k+1}}}
\end{aligned}
$$

for $i \in\{1, \ldots, n\}$. Equivalently, (31) can be written in a matrix form as

$$
\begin{gathered}
{\left[\begin{array}{cccc}
M_{11} I_{3 \times 3} & -M_{12} \hat{q}_{1_{k+1}} \hat{q}_{2_{k+1}} & \cdots & -M_{1 n} \hat{q}_{1} \hat{q}_{n_{k+1}} \\
-M_{21} \hat{q}_{2_{k+1}} \hat{q}_{1_{k+1}} & M_{22} I_{3 \times 3} & \cdots & -M_{2 n} \hat{q}_{2_{k+1}} \hat{q}_{n_{k+1}} \\
\vdots & \vdots & & \vdots \\
-M_{n 1} \hat{q}_{n_{k+1}} \hat{q}_{1_{k+1}} & -M_{n 2} \hat{q}_{n_{k+1}} \hat{q}_{2_{k+1}} & \cdots & M_{n n} I_{3 \times 3}
\end{array}\right]\left[\begin{array}{c}
\omega_{1_{k+1}} \\
\omega_{2_{k+1}} \\
\vdots \\
\omega_{n_{k+1}}
\end{array}\right]} \\
=\left[\begin{array}{c}
\frac{1}{h}\left(q_{1_{k+1}} \times \sum_{j=1}^{n} M_{1 j}\left(q_{j_{k+1}}-q_{j_{k}}\right)\right)-\frac{h}{2} q_{1_{k+1}} \times \frac{\partial V_{k+1}}{\partial q_{1_{k+1}}} \\
\frac{1}{h}\left(q_{2_{k+1}} \times \sum_{j=1}^{n} M_{2 j}\left(q_{j_{k+1}}-q_{j_{k}}\right)\right)-\frac{h}{2} q_{2_{k+1}} \times \frac{\partial V_{k+1}}{\partial q_{2_{k+1}}} \\
\vdots \\
\frac{1}{h}\left(q_{n_{k+1}} \times \sum_{j=1}^{n} M_{n j}\left(q_{j_{k+1}}-q_{j k}\right)\right)-\frac{h}{2} q_{n_{k+1}} \times \frac{\partial V_{k+1}}{\partial q_{n_{k+1}}}
\end{array}\right]
\end{gathered}
$$

For a given $\left(q_{i_{k}}, \omega_{i_{k}}\right)$, we solve (29) to obtain $F_{i_{k}} \in \mathrm{SO}(3)$. Then, $q_{i_{k+1}}$ and $\omega_{i_{k+1}}$ are computed by (30) and (32), respectively. This yields a discrete flow map in terms of the angular velocity $\left(q_{i_{k}}, \omega_{i_{k}}\right) \mapsto\left(q_{i_{k+1}}, \omega_{i_{k+1}}\right)$ and this process is repeated. 


\subsection{Computational approach}

For the discrete equations of motion, we need to solve (21) and (29) to obtain $F_{i_{k}} \in \mathrm{SO}(3)$. Here we present a computational approach. The implicit equations given by (21) and (29) have the following structure:

$$
M_{i i} q_{i} \times F_{i} q_{i}+q_{i} \times \sum_{\substack{j=1 \\ j \neq i}}^{n} M_{i j}\left(F_{j}-I_{3 \times 3}\right) q_{j}=d_{i}
$$

for $i \in\{1, \ldots, n\}$, where $M_{i j} \in \mathbb{R}, q_{i} \in \mathbb{S}^{2}, d_{i} \in \mathbb{R}^{3}$ are known, and we need to find $F_{i} \in \operatorname{SO}(3)$. We derive an equivalent equation in terms of local coordinates for $F_{i}$. This is reasonable since $F_{i}$ represents the relative update between two integration steps. Using the Cayley transformation [11], $F_{i} \in \mathrm{SO}(3)$ can be expressed in terms of $f_{i} \in \mathbb{R}^{3}$ as

$$
F_{i}=\left(I_{3 \times 3}+\hat{f}_{i}\right)\left(I_{3 \times 3}-\hat{f}_{i}\right)^{-1}=\frac{1}{1+f_{i} \cdot f_{i}}\left(\left(1-f_{i} \cdot f_{i}\right) I_{3 \times 3}+2 f_{i} f_{i}^{\mathrm{T}}+2 \hat{f}_{i}\right)
$$

The operation $F_{i} q_{i}$ can be considered as a rotation of the vector $q_{i}$ about the direction $f_{i}$ with rotation angle $2 \tan ^{-1}\left\|f_{i}\right\|$. Since the rotation of the vector $q_{i}$ about the direction $q_{i}$ has no effect, we can assume that $f_{i}$ is orthogonal to $q_{i}$, i.e. $f_{i} \cdot q_{i}=0$. Under this assumption, $F_{i} q_{i}$ is given by

$$
F_{i} q_{i}=\frac{1}{1+f_{i} \cdot f_{i}}\left(\left(1-f_{i} \cdot f_{i}\right) q_{i}+2 \hat{f}_{i} q_{i}\right)
$$

Thus, we obtain

$$
\begin{aligned}
q_{i} \times F_{i} q_{i} & =\frac{2}{1+f_{i} \cdot f_{i}} q_{i} \times\left(f_{i} \times q_{i}\right)=\frac{2}{1+f_{i} \cdot f_{i}} f_{i} \\
\left(F_{j}-I_{3 \times 3}\right) q_{j} & =-\frac{2}{1+f_{j} \cdot f_{j}}\left(q_{j} f_{j}^{\mathrm{T}}+\hat{q}_{j}\right) f_{j}
\end{aligned}
$$

where we use the property, $\hat{q}_{i} f_{i}=q_{i} \times f_{i}=-\hat{f}_{i} q_{i}$. Substituting these into (33), we obtain

$$
\begin{gathered}
{\left[\begin{array}{cccc}
\frac{2 M_{11} I_{3 \times 3}}{1+f_{1} \cdot f_{1}} & -\frac{2 M_{12} \hat{q}_{1}\left(\hat{q}_{2}+q_{2} f_{2}^{\mathrm{T}}\right)}{1+f_{2} \cdot f_{2}} & \ldots & -\frac{2 M_{1 n} \hat{q}_{1}\left(\hat{q}_{n}+q_{n} f_{n}^{\mathrm{T}}\right)}{1+f_{n} \cdot f_{n}} \\
-\frac{2 M_{21} \hat{q}_{2}\left(\hat{q}_{1}+q_{1} f_{1}^{\mathrm{T}}\right)}{1+f_{1} \cdot f_{1}} & \frac{2 M_{22} I_{3 \times 3}}{1+f_{2} \cdot f_{2}} & \ldots & -\frac{2 M_{2 n} \hat{q}_{2}\left(\hat{q}_{n}+q_{n} f_{n}^{\mathrm{T}}\right)}{1+f_{n} \cdot f_{n}} \\
\vdots & \vdots & \vdots \\
-\frac{2 M_{n 1} \hat{q}_{n}\left(\hat{q}_{1}+q_{1} f_{1}^{\mathrm{T}}\right)}{1+f_{1} \cdot f_{1}} & -\frac{2 M_{n 2} \hat{q}_{n}\left(\hat{q}_{2}+q_{2} f_{2}^{\mathrm{T}}\right)}{1+f_{2} \cdot f_{2}} & \ldots & \frac{2 M_{n n} I_{3 \times 3}}{1+f_{n} \cdot f_{n}}
\end{array}\right]\left[\begin{array}{c}
f_{1} \\
f_{2} \\
\vdots \\
f_{n}
\end{array}\right]} \\
=\left[\begin{array}{c}
d_{1} \\
d_{2} \\
\vdots \\
d_{n}
\end{array}\right]
\end{gathered}
$$


which is an equation equivalent to (33), written in terms of local coordinates for $F_{i}$ using the Cayley transformation. Any numerical method to solve non-linear equations can be applied to find $f_{i}$. Then, $F_{i} q_{i}$ is computed by using (34). In particular, (35) is written in a form that can be readily applied to a fixed point iteration method [18].

If there are no coupling terms in the kinetic energy, we can obtain an explicit solution of (33). When $M_{i j}=0$ for $i \neq j$, (35) reduces to

$$
\frac{2 M_{i i}}{1+f_{i} \cdot f_{i}} f_{i}=d_{i}
$$

Using the identity, $2 \tan \theta /\left(1+\tan ^{2} \theta\right)=\sin 2 \theta$ for any $\theta \in \mathbb{R}$, it can be shown that the solution of this equation is given by $f_{i}=\tan \left(\frac{1}{2} \sin ^{-1}\left(\left\|d_{i}\right\| / M_{i i}\right)\right) \frac{d_{i}}{\left\|d_{i}\right\|}$. Substituting this into (34) and rearranging, we obtain

$$
F_{i} q_{i}=\frac{d_{i}}{M_{i i}} \times q_{i}+\left(1-\left\|\frac{d_{i}}{M_{i i}}\right\|^{2}\right)^{1 / 2} q_{i}
$$

Using this expression, we can rewrite the discrete equations of motion given in (29)-(32) in an explicit form.

\section{Corollary 5}

Consider a mechanical system on $\left(\mathbb{S}^{2}\right)^{n}$ whose Lagrangian is expressed as (2) where $M_{i j}=0$ for $i \neq j$, i.e. the dynamics are coupled only though the potential energy. The explicit discrete equations of motion are given by

$$
\begin{aligned}
q_{i_{k+1}} & =\left(h \omega_{i_{k}}-\frac{h^{2}}{2 M_{i i}} q_{i_{k}} \times \frac{\partial V_{k}}{\partial q_{i_{k}}}\right) \times q_{i_{k}}+\left(1-\left\|h \omega_{i_{k}}-\frac{h^{2}}{2 M_{i i}} q_{i_{k}} \times \frac{\partial V_{k}}{\partial q_{i_{k}}}\right\|^{2}\right)^{1 / 2} q_{i_{k}} \\
\omega_{i_{k+1}} & =\omega_{i_{k}}-\frac{h}{2 M_{i i}} q_{i_{k}} \times \frac{\partial V_{k}}{\partial q_{i_{k}}}-\frac{h}{2 M_{i i}} q_{i_{k+1}} \times \frac{\partial V_{k+1}}{\partial q_{i_{k+1}}}
\end{aligned}
$$

for $i \in\{1, \ldots, n\}$.

\subsection{Properties of variational integrators on $\left(\mathbb{S}^{2}\right)^{n}$}

Since variational integrators are derived from the discrete Hamilton's principle, they are symplectic and momentum preserving. The discrete action sum can be considered as a zero-form on $\left(\mathbb{S}^{2}\right)^{n} \times$ $\left(\mathbb{S}^{2}\right)^{n}$ that maps the initial condition of a discrete flow satisfying the discrete Euler-Lagrange equation to the action sum for that trajectory. The simplicity of the discrete flow follows from the fact the iterated exterior derivative of any differential form is zero. If the discrete Lagrangian exhibits a symmetry, the corresponding momentum map is preserved since by symmetry, the variation of the discrete Lagrangian in the symmetry direction is zero, which in combination with the discrete Euler-Lagrange equations implies a discrete version of Noether's theorem. Detailed proofs for the symplectic property and the momentum preserving property can be found in [6]. The total energy oscillates around its initial value with small bounds on a comparatively short timescale, but there is no tendency for the mean of the oscillation in the total energy to drift (increase or decrease) over exponentially long times [19]. 
The variational integrators presented in this paper preserve the structure of $\left(\mathbb{S}^{2}\right)^{n}$ without need of local parameterizations, explicit constraints, or reprojection. Using the characteristics of the homogeneous manifold, the discrete update map is represented by a group action of $\mathrm{SO}(3)$, and a proper subspace is searched to obtain a compact, possibly explicit form for the numerical integrator. As a result, the following numerical problems are avoided: (i) local parameterizations yield singularities, (ii) numerical trajectories in the vicinity of a singularity experience numerical ill-conditioning, (iii) unit length constraints lead to additional computational complexity, and (iv) reprojection corrupts the numerical accuracy of trajectories $[3,9]$.

It can be shown that these variational integrators have second-order accuracy as the discrete action sum is a second-order approximation of the action integral, which we have constructed through linear interpolation and the trapezoidal approximation of the integral. Indeed, the variational integrator obtained for $\left(\mathbb{S}^{2}\right)^{n}$ for this discrete Lagrangian is equivalent to the RATTLE discretization [20] of the corresponding constrained Lagrangian system with the Lagrange multipliers explicitly eliminated. More formally, one can consider the construction we have presented in this paper to be a systematic means of constructing a symplectic integrator based on indexreduction of the constraints. While higher-order integrators can be easily constructed by applying a symmetric composition method [21], this work also provides a framework for directly obtaining higher-order variational integrators for Lagrangian flows on $\left(\mathbb{S}^{2}\right)^{n}$.

\subsection{Numerical examples}

The computational properties of variational integrators on $\left(\mathbb{S}^{2}\right)^{n}$ and explicit Runge-Kutta methods are compared for several mechanical systems taken from variety of scientific areas, namely a double spherical pendulum, an $n$-body problem on a sphere, an interconnected system of spherical pendula, pure bending of a geometrically-exact elastic rod, a spatial array of magnetic dipoles, and molecular dynamics that evolves on a sphere. All of the computational results in this section are obtained by Matlab.

\section{Example 1 (double spherical pendulum)}

A double spherical pendulum is defined by two mass particles serially connected to frictionless two DOF pivots by rigid massless links acting under a uniform gravitational potential. The dynamics of a double spherical pendulum has been studied in [2], and a variational integrator is developed in [22] by explicitly using unit length constraints.

Let the mass and the length of the pendulum be $m_{1}, m_{2}, l_{1}, l_{2} \in \mathbb{R}$, respectively and let $e_{3}=$ $[0,0,1] \in \mathbb{R}^{3}$ be the direction of gravity. The vector $q_{1} \in \mathbb{S}^{2}$ represents the direction from the pivot to the first mass and the vector $q_{2} \in \mathbb{S}^{2}$ represents the direction from the first mass to the second mass. The inertia matrix is given by $M_{11}=\left(m_{1}+m_{2}\right) l_{1}^{2}, M_{12}=m_{2} l_{1} l_{2}$, and $M_{22}=m_{2} l_{2}^{2}$. The gravitational potential is written as $V\left(q_{1}, q_{2}\right)=-\left(m_{1}+m_{2}\right) g l_{1} e_{3} \cdot q_{1}-m_{2} g l_{2} e_{3} \cdot q_{2}$ for the gravitational acceleration $g \in \mathbb{R}$. Substituting these into (10)-(11), the continuous equations of motion for the double spherical pendulum are given by

$$
\begin{aligned}
& \dot{q}_{1}=\omega_{1} \times q_{1}, \quad \dot{q}_{2}=\omega_{2} \times q_{2} \\
& {\left[\begin{array}{cc}
\left(m_{1}+m_{2}\right) l_{1}^{2} I_{3 \times 3} & -m_{2} l_{1} l_{2} \hat{q}_{1} \hat{q}_{2} \\
-m_{2} l_{1} l_{2} \hat{q}_{2} \hat{q}_{1} & m_{2} l_{2}^{2} I_{3 \times 3}
\end{array}\right]\left[\begin{array}{c}
\dot{\omega}_{1} \\
\dot{\omega}_{2}
\end{array}\right]=\left[\begin{array}{c}
m_{2} l_{1} l_{2}\left(\omega_{2} \cdot \omega_{2}\right) \hat{q}_{1} q_{2}+\left(m_{1}+m_{2}\right) g l_{1} \hat{q}_{1} e_{3} \\
m_{2} l_{1} l_{2}\left(\omega_{1} \cdot \omega_{1}\right) \hat{q}_{2} q_{1}+m_{2} g l_{2} \hat{q}_{2} e_{3}
\end{array}\right] }
\end{aligned}
$$


(a)
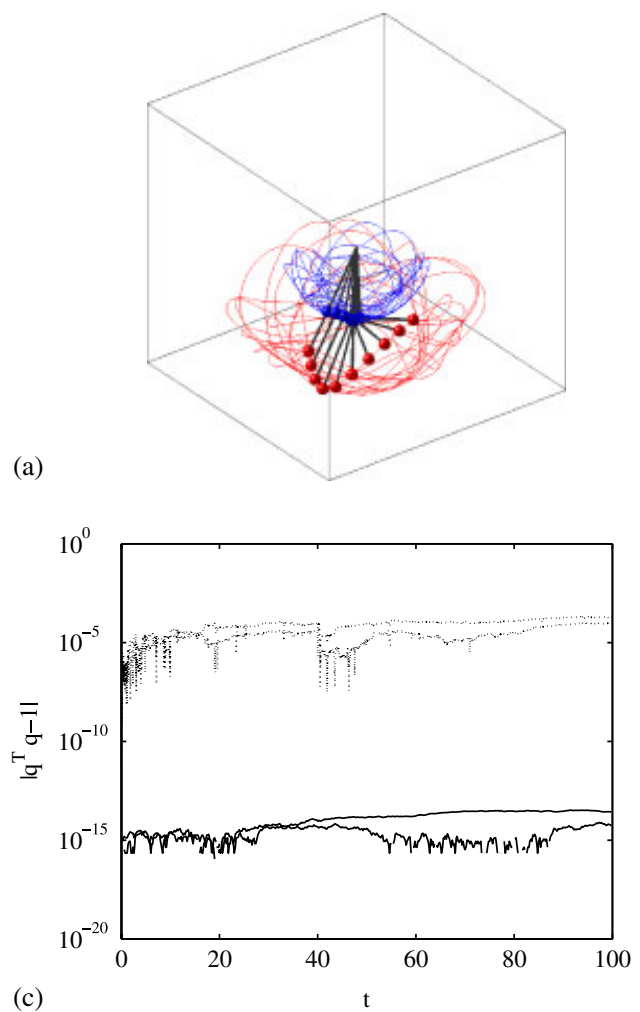

(b)
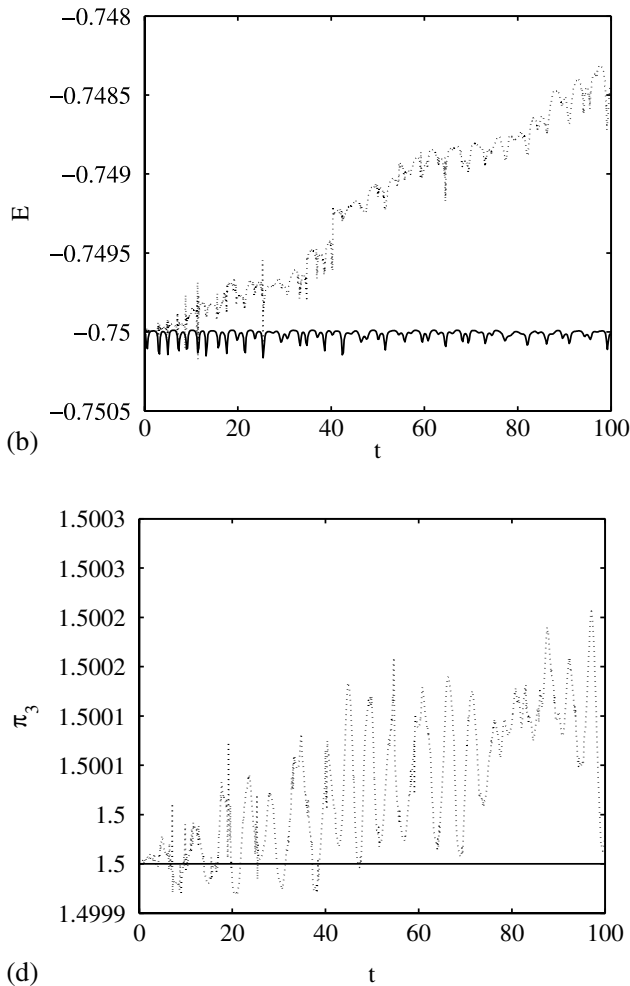

Figure 1. Numerical simulation of a double spherical pendulum (RK45: dotted, VI: solid): (a) trajectory of pendulum; (b) computed total energy; (c) unit length error; and (d) angular momentum about the vertical axis.

which are more compact than the existing equations written in terms of angles. Another nice property is that the same structure for the equations of motion is maintained for $n>2$. Thus, it is easy to generalize these equations of motion to a triple, or more generally, a multiple-link spherical pendulum.

We compare the computational properties of the discrete equations of motion given by (29)-(32) with a 4(5)th-order variable step size Runge-Kutta method, implemented by the Matlab ode45 function, for (38)-(39). We choose $m_{1}=m_{2}=1 \mathrm{~kg}, l_{1}=l_{2}=9.81 \mathrm{~m}$. The initial conditions are $q_{1_{0}}=[0.8660,0,0.5], q_{2_{0}}=[0,0,1], \omega_{1_{0}}=[-0.4330,0,0.75], \omega_{2_{0}}=[0,1,0] \mathrm{rad} / \mathrm{s}$. The simulation time is $100 \mathrm{~s}$ and the step-size of the discrete equations of motion is $h=0.01$. Figure 1 shows the computed total energy, the configuration manifold errors, and the computed total angular momentum about the vertical $e_{3}$ axis. The variational integrator preserves these conserved quantities well for this chaotic motion of the double spherical pendulum. The mean total energy variation is $2.1641 \times 10^{-5} \mathrm{Nm}$, the mean unit length error is $8.8893 \times 10^{-15}$, and the mean angular momentum error is $3.6361 \times 10^{-11} \mathrm{Nms}$, But, there is a notable increase of the computed total energy for the Runge-Kutta method, where the mean variation of the total energy is $7.8586 \times 10^{-4} \mathrm{Nm}$. The Runge-Kutta method also fails to preserve the structure of $\left(\mathbb{S}^{2}\right)^{n}$ and the angular momentum. 
The mean unit length error and the mean angular momentum conservation error are $6.2742 \times 10^{-5}$ and $8.2068 \times 10^{-5} \mathrm{Nms}$, respectively.

\section{Example 2 (n-body problem on sphere)}

An $n$-body problem on the two-sphere deals with the motion of $n$ mass particles constrained to lie on a two-sphere, acting under a mutual potential. Let $m_{i} \in \mathbb{R}$ and $q_{i} \in \mathbb{S}^{2}$ be the mass and the position vector of the $i$ th particle, respectively. The $i, j$ th element of the inertia matrix is $M_{i j}=m_{i}$ when $i=j$ and $M_{i j}=0$ otherwise. In [23], the following expression for the potential is introduced as an analog of a gravitational potential:

$$
V\left(q_{1}, \ldots, q_{n}\right)=-\frac{\gamma}{2} \sum_{\substack{i, j=1 \\ i \neq j}}^{n} \frac{q_{i} \cdot q_{j}}{\sqrt{1-\left(q_{i} \cdot q_{j}\right)^{2}}}
$$

for a constant $\gamma$. Substituting these into (7), the continuous equations of motion for the $n$-body problem on a sphere are given by

$$
m_{i} \ddot{q}_{i}=-m_{i}\left(\dot{q}_{i} \cdot \dot{q}_{i}\right) q_{i}-q_{i} \times\left(q_{i} \times \gamma \sum_{\substack{j=1 \\ j \neq i}}^{n} \frac{q_{j}}{\left(1-\left(q_{i} \cdot q_{j}\right)^{2}\right)^{3 / 2}}\right)
$$

for $i \in\{1, \ldots, n\}$.

A two-body problem on the two-sphere under this gravitational potential is studied in [24] by explicitly using unit length constraints. Here we study a three-body problem, $n=3$. Since there are no coupling terms in the kinetic energy, we use the explicit form of the variational integrator. We compare the computational properties of the discrete equations of motion given by (36)-(37) with a second-order fixed step size Runge-Kutta method for (40). We choose $m_{1}=m_{2}=m_{3}=1$ and $\gamma=1$. The initial conditions are $q_{1_{0}}=[0,-1,0], q_{2_{0}}=[0,0,1], q_{3_{0}}=[-1,0,0], \omega_{1_{0}}=[0,0,-1.1]$, $\omega_{2_{0}}=[1,0,0]$, and $\omega_{3_{0}}=[0,1,0]$. The simulation time is $10 \mathrm{~s}$. Figure 2 shows the computed total energy and the unit length errors for various step sizes. The total energy variations and the unit length errors for the variational integrator are smaller than those of the Runge-Kutta method for the same time step size by several orders of magnitude. For the variational integrator, the total energy error is reduced by almost 100 times from $1.1717 \times 10^{-4}$ to $1.1986 \times 10^{-6}$ when the step size is reduced by 10 times from $10^{-3}$ to $10^{-4}$, which verifies the second-order accuracy numerically.

Example 3 (interconnection of spherical pendula)

We study the dynamics of $n$ spherical pendula connected by linear springs. Each pendulum is a mass particle connected to a frictionless two DOF pivot by a rigid massless link acting under a uniform gravitational potential. It is assumed that all of the pivot points lie on a horizontal plane and some pairs of pendula are connected by linear springs at the centers of links.

Let the mass and the length of the $i$ th pendulum be $m_{i}, l_{i} \in \mathbb{R}$, respectively. The vector $q_{i} \in \mathbb{S}^{2}$ represents the direction from the $i$ th pivot to the $i$ th mass. The inertia matrix is given by $M_{i j}=m_{i} l_{i}^{2}$ when $i=j$ and $M_{i j}=0$ otherwise. Let $\Xi$ be a set defined such that $(i, j) \in \Xi$ if the $i$ th pendulum and the $j$ th pendulum are connected. For a connected pair $(i, j) \in \Xi$, define $\kappa_{i j} \in \mathbb{R}$ and $r_{i j} \in \mathbb{R}^{3}$ as the corresponding spring constant and the vector from the $i$ th pivot to the $j$ th pivot, respectively. The bases for the inertial frame are chosen such that the direction along gravity is denoted 


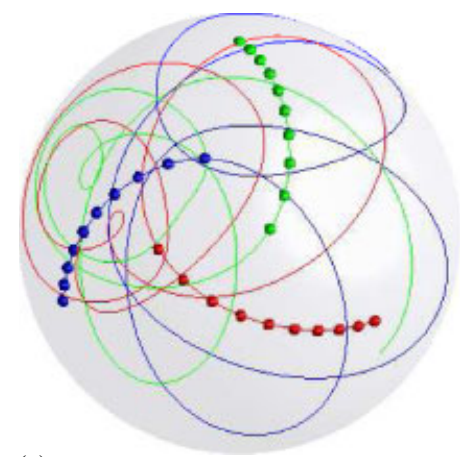

(a)

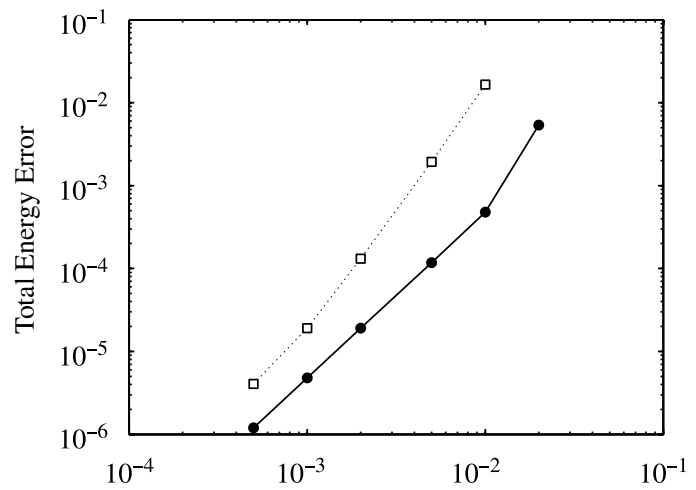

(b)

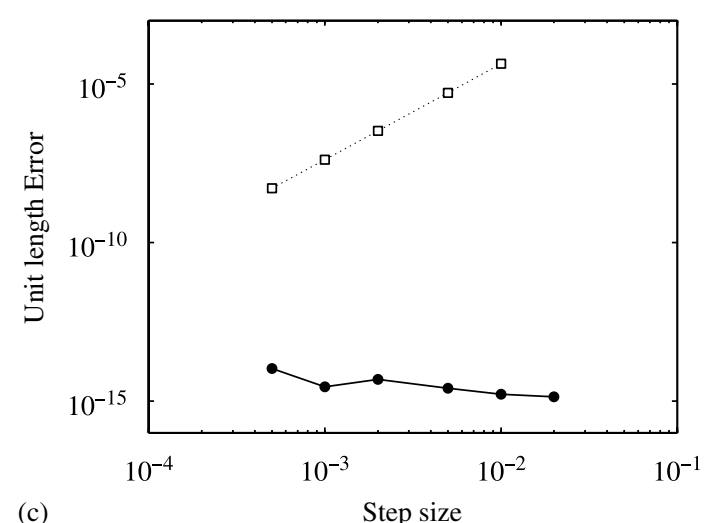

(c)

Figure 2. Numerical simulation of a 3-body problem on sphere (RK2: square, dotted, VI: circle, solid): (a) trajectory of particles; (b) total energy error vs step size; and (c) unit length error vs step size.

by $e_{3}=[0,0,1] \in \mathbb{R}^{3}$ and the horizontal plane is spanned by $e_{1}=[0,0,1], e_{2}=[0,1,0] \in \mathbb{R}^{3}$. The potential energy is given by

$$
V\left(q_{1}, \ldots, q_{n}\right)=-\sum_{i=1}^{n} m_{i} g l_{i} q_{i} \cdot e_{3}+\sum_{(i, j) \in \Xi} \frac{1}{2} \kappa_{i j}\left(\left\|r_{i j}+\frac{1}{2} l_{j} q_{j}-\frac{1}{2} l_{i} q_{i}\right\|-\left\|r_{i j}\right\|\right)^{2}
$$

Substituting these into (9)-(10), the continuous equations of motion for the interconnection of spherical pendula are given by

$$
\begin{aligned}
m_{i} l_{i}^{2} \dot{\omega}_{i} & =-q_{i} \times \frac{\partial V}{\partial q_{i}} \\
\dot{q}_{i} & =\omega_{i} \times q_{i}
\end{aligned}
$$

for $i \in\{1, \ldots, n\}$.

We compare the computational properties of the discrete equations of motion given by (36)-(37) with a second-order fixed step size explicit Runge-Kutta method for (41)-(42) and the same Runge-Kutta method with reprojection; at each time step, the vectors $q_{i_{k}}$ are projected onto $\mathbb{S}^{2}$ by using normalization. 

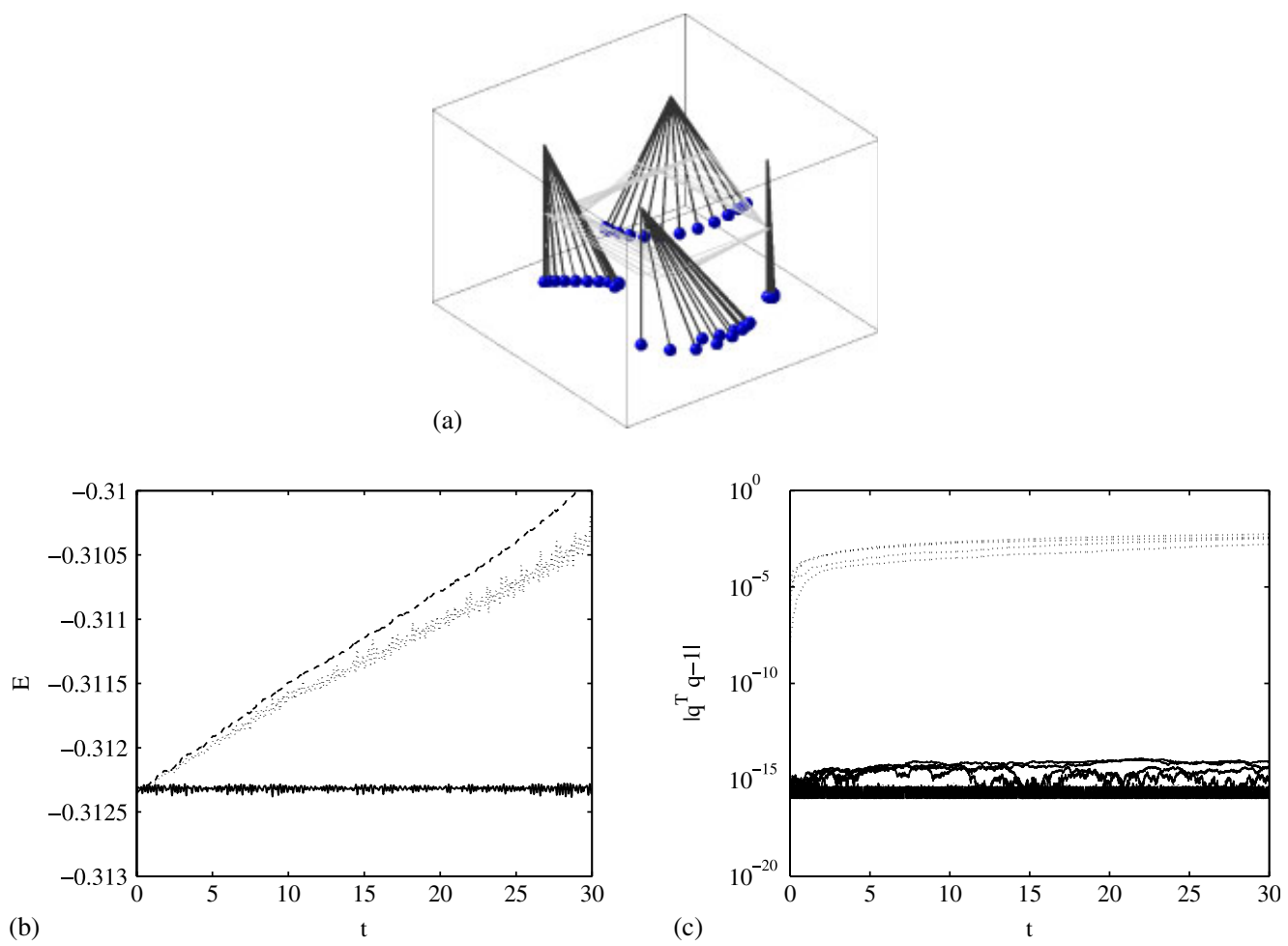

Figure 3. Numerical simulation of a system of four spherical pendula (RK2: dotted, RK2 with projection: dashed, VI: solid): (a) motion of pendula; (b) computed total energy; and (c) unit length error.

We choose four interconnected pendula, $n=4$, and we assume each pendulum has the same mass and length; $m_{i}=0.1 \mathrm{~kg}, l_{i}=0.1 \mathrm{~m}$. The pendula are connected as $\Xi=\{(1,2),(2,3),(3,4),(4,1)\}$ and the corresponding spring constants and the relative vector between pivots are given by $\kappa_{12}=10, \kappa_{12}=20, \kappa_{12}=30, \kappa_{12}=40 \mathrm{~N} / \mathrm{m}, r_{12}=-r_{34}=l_{i} e_{1}$, and $r_{23}=-r_{41}=-l_{i} e_{2}$. The initial conditions are chosen as $q_{1_{0}}=q_{2_{0}}=q_{4_{0}}=e_{3}, q_{3_{0}}=[0.4698,0.1710,0.8660], \omega_{1_{0}}=[-10,4,0]$, and $\omega_{2_{0}}=\omega_{3_{0}}=\omega_{4_{0}}=0 \mathrm{rad} / \mathrm{s}$.

Figure 3 shows the computed total energy and the unit length errors. The variational integrator preserves the total energy and the structure of $\left(\mathbb{S}^{2}\right)^{n}$ well. The mean total energy variation is $3.6171 \times 10^{-5} \mathrm{Nm}$, and the mean unit length error is $4.2712 \times 10^{-15}$. For both Runge-Kutta methods, there is a notable increase of the computed total energy. It is interesting to see that the reprojection approach makes the total energy error worse, even though it preserves the structure of $\left(\mathbb{S}^{2}\right)^{n}$ accurately. This shows that a standard reprojection method can corrupt numerical trajectories $[3,9]$.

\section{Example 4 (pure bending of geometrically exact elastic rod)}

We study the dynamics of $(n+1)$ rigid rod elements that are serially connected by rotational springs, where the 'zeroth' rod is assumed to be fixed to a wall. Thus, the configuration space is $\left(\mathbb{S}^{2}\right)^{n}$. This can be considered as a simplified dynamics model for pure nonplanar bending of a thin elastic 
rod that is clamped at one end and free at the other end. Notably, this approach is geometrically exact and preserves the length of the elastic rod in the presence of large displacements.

The mass and the length of the $i$ th rod element are denoted by $m_{i}, l_{i} \in \mathbb{R}$, respectively. The inertia matrix is given by

$$
M_{i i}=\frac{1}{3} m_{i} l_{i}^{2}+\sum_{k=i+1}^{n} m_{i} l_{i}^{2}, \quad M_{i j}=\sum_{k=\max \{i, j\}}^{n} \frac{1}{2} m_{k} l_{k}^{2}
$$

for $i, j \in\{1, \ldots, n\}$ and $i \neq j$. The potential energy is composed of gravitational terms and elastic bending terms given by

$$
V\left(q_{1}, \ldots, q_{n}\right)=-\sum_{i=1}^{n} m_{i} g\left(\sum_{j=1}^{i-1} l_{j} q_{j}+\frac{1}{2} l_{i} q_{i}\right) \cdot e_{3}+\frac{1}{2} \kappa_{i}\left(1-q_{i-1} \cdot q_{i}\right)^{2}
$$

where a constant vector $q_{0} \in \mathbb{S}^{2}$ denotes the direction of the zeroth rod element fixed to a wall and $\kappa_{i} \in \mathbb{R}$ denotes spring constants. The bases for the inertial frame are chosen such that the gravity direction is denoted by $e_{3}=[0,0,1] \in \mathbb{R}^{3}$ and the horizontal plane is spanned by $e_{1}=[0,0,1], e_{2}=$ $[0,1,0] \in \mathbb{R}^{3}$. Suppose that the total mass and length of rod are given by $m, l$ and each rod element has the same mass and length, i.e. $m_{i}=m /(n+1), l_{i}=l /(n+1)$ for $i \in\{0, \ldots, n\}$. Substituting these into (8), the continuous equations of motion for the pure bending of an elastic rod are given by

$$
\begin{aligned}
& {\left[\begin{array}{cccc}
\frac{n-2 / 3}{(n+1)^{3}} m l^{2} I_{3 \times 3} & -\frac{n-1}{2(n+1)^{3}} m l^{2} \hat{q}_{1} \hat{q}_{1} & \ldots & -\frac{1}{2(n+1)^{3}} m l^{2} \hat{q}_{1} \hat{q}_{1} \\
-\frac{n-1}{2(n+1)^{3}} m l^{2} \hat{q}_{2} \hat{q}_{2} & \frac{n-5 / 3}{(n+1)^{3}} m l^{2} I_{3 \times 3} & \ldots & -\frac{1}{2(n+1)^{3}} m l^{2} \hat{q}_{2} \hat{q}_{2} \\
\vdots & \vdots \\
-\frac{1}{2(n+1)^{3}} m l^{2} \hat{q}_{n} \hat{q}_{n} & -\frac{1}{2(n+1)^{3}} m l^{2} \hat{q}_{n} \hat{q}_{n} & \ldots & \frac{1 / 3}{(n+1)^{3}} m l^{2} I_{3 \times 3}
\end{array}\right]\left[\begin{array}{c}
\ddot{q}_{1} \\
\ddot{q}_{2} \\
\vdots \\
\ddot{q}_{n}
\end{array}\right]} \\
& =\left[\begin{array}{c}
-\frac{n-2 / 3}{(n+1)^{3}} m l^{2}\left(\dot{q}_{1} \cdot \dot{q}_{1}\right) q_{1}+\hat{q}_{1}^{2} \frac{\partial V}{\partial q_{1}} \\
-\frac{n-5 / 3}{(n+1)^{3}} m l^{2}\left(\dot{q}_{2} \cdot \dot{q}_{2}\right) q_{2}+\hat{q}_{2}^{2} \frac{\partial V}{\partial q_{2}} \\
\vdots \\
-\frac{1 / 3}{(n+1)^{3}} m l^{2}\left(\dot{q}_{n} \cdot \dot{q}_{n}\right) q_{n}+\hat{q}_{n}^{2} \frac{\partial V}{\partial q_{n}}
\end{array}\right]
\end{aligned}
$$

We compare the computational properties of the discrete equations of motion given by (29)-(32) with a 4(5)th-order variable step size Runge-Kutta method, implemented by the Matlab ode45 function, for (43). We choose 10 rod elements, $n=10$, and the total mass and the total length are $m=55 \mathrm{~g}, l=1.1 \mathrm{~m}$. The spring constants are chosen as $\kappa_{i}=1000 \mathrm{Nm}$. Initially, the rod is aligned horizontally; $q_{i_{0}}=e_{1}$ for all $i \in 1, \ldots, n$. The initial angular velocity for each rod element is zero 


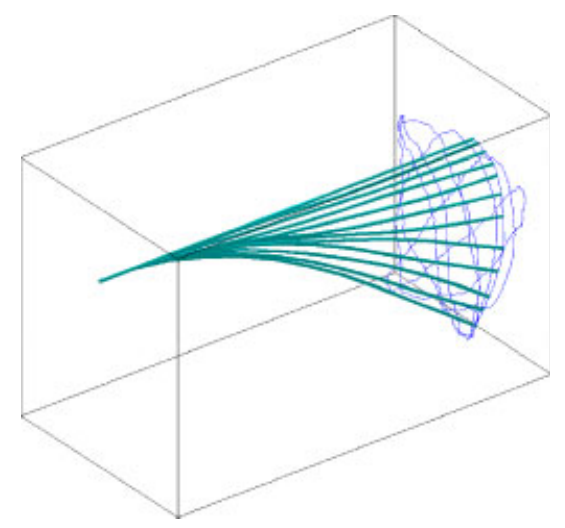

(a)
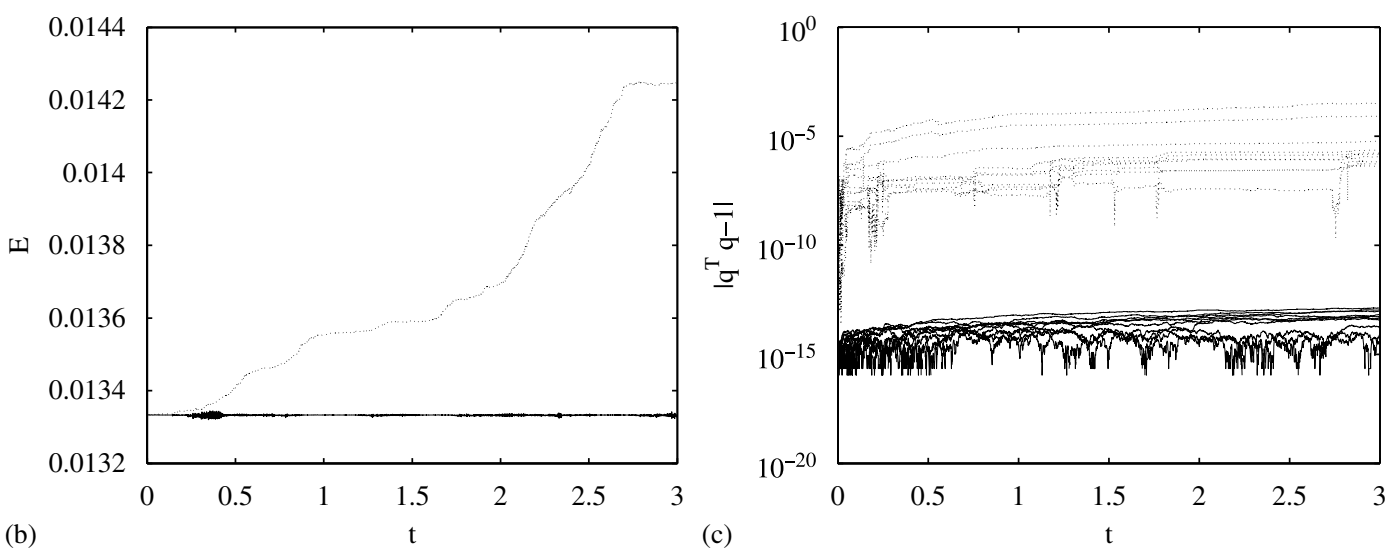

Figure 4. Numerical simulation of an elastic rod (RK45: dotted, VI: solid): (a) deformation of rod; (b) computed total energy; and (c) unit length error.

except $\omega_{5_{0}}=[0,0,10] \mathrm{rad} / \mathrm{s}$. This represents the dynamics of the rod after an initial impact. The simulation time is $3 \mathrm{~s}$ and the step size of the discrete equations of motion is $h=0.0001$.

Figure 4 shows the computed total energy and the unit length errors. The variational integrator preserves the total energy and the structure of $\left(\mathbb{S}^{2}\right)^{n}$. The mean total energy variation is $1.4310 \times$ $10^{-6} \mathrm{Nm}$ and the mean unit length error is $2.9747 \times 10^{-14}$. There is a notable dissipation of the computed total energy for the Runge-Kutta method, where the mean variation of the total energy is $3.5244 \times 10^{-4} \mathrm{Nm}$. The Runge-Kutta method also fails to preserve the structure of $\left(\mathbb{S}^{2}\right)^{n}$. The mean unit length error is $1.8725 \times 10^{-5}$.

Example 5 (spatial array of magnetic dipoles)

We study dynamics of $n$ magnetic dipoles uniformly distributed on a plane. Each magnetic dipole is modeled as a spherical compass; a thin rod magnet supported by a frictionless, two DOF pivot acting under their mutual magnetic field. This can be considered as a simplified model for the dynamics of micromagnetic particles [25]. 
The mass and the length of the $i$ th magnet are denoted by $m_{i}, l_{i} \in \mathbb{R}$, respectively. The magnetic dipole moment of the $i$ th magnet is denoted by $v_{i} q_{i}$, where $v_{i} \in \mathbb{R}$ is the constant magnitude of the magnetic moment measured in ampere square-meters and $q_{i} \in \mathbb{S}^{2}$ is the direction of the north pole from the pivot point. Thus, the configuration space is $\left(\mathbb{S}^{2}\right)^{n}$. The inertia matrix is given by $M_{i j}=\frac{1}{12} m_{i} l_{i}^{2}$ when $i=j$ and $M_{i j}=0$ otherwise. Let $r_{i j} \in \mathbb{R}^{3}$ be the vector from the $i$ th pivot point to the $j$ th pivot point. The mutual potential energy of the array of magnetic dipoles is given by

$$
V\left(q_{1}, \ldots, q_{n}\right)=\frac{1}{2} \sum_{\substack{i, j=1 \\ j \neq i}}^{n} \frac{\mu v_{i} v_{j}}{4 \pi\left\|r_{i j}\right\|^{3}}\left[\left(q_{i} \cdot q_{j}\right)-\frac{3}{\left\|r_{i j}\right\|^{2}}\left(q_{i} \cdot r_{i j}\right)\left(q_{j} \cdot r_{i j}\right)\right]
$$

where $\mu=4 \pi \times 10^{-7} \mathrm{NA}^{-2}$ is the permeability constant. Substituting these into (9)-(10), the continuous equations of motion for the spatial array of magnetic dipoles are given by

$$
\begin{aligned}
\frac{1}{12} m_{i} l_{i}^{2} \dot{\omega}_{i} & =-q_{i} \times \sum_{\substack{j=1 \\
j \neq i}}^{n} \frac{\mu v_{i} v_{j}}{4 \pi\left\|r_{i j}\right\|^{3}}\left[q_{j}-\frac{3}{\left\|r_{i j}\right\|^{2}} r_{i j}\left(q_{j} \cdot r_{i j}\right)\right] \\
\dot{q}_{i} & =\omega_{i} \times q_{i}
\end{aligned}
$$

for $i \in\{1, \ldots, n\}$.

We compare the computational properties of the discrete equations of motion given by (36)-(37) with a 4(5)th-order variable step size Runge-Kutta method, implemented by the Matlab ode45 function, for (44)-(45). We choose 16 magnetic dipoles, $n=16$, and we assume each magnetic dipole has the same mass, length, and magnitude of magnetic moment; $m_{i}=0.05 \mathrm{~kg}, l_{i}=0.02 \mathrm{~m}$, $v_{i}=0.1 \mathrm{Am}^{2}$. The magnetic dipoles are located at vertices of a $4 \times 4$ square grid in which the edge of a unit square has the length of $1.2 l_{i}$. The initial conditions are chosen as $q_{i_{0}}=[1,0,0], \omega_{i_{0}}=[0,0,0]$ for all $i \in\{1, \ldots, 16\}$ except $q_{16_{0}}=[0.3536,0.3536,-0.8660]$ and $\omega_{1_{0}}=[0,0.5,0] \mathrm{rad} / \mathrm{s}$.

Figure 5 shows the computed total energy and the unit length errors. The variational integrator preserves the total energy and the structure of $\left(\mathbb{S}^{2}\right)^{n}$ well. The mean total energy variation is $8.5403 \times 10^{-10} \mathrm{Nm}$ and the mean unit length error is $1.6140 \times 10^{-14}$. There is a notable dissipation of the computed total energy for the Runge-Kutta method, where the mean variation of the total energy is $2.9989 \times 10^{-7} \mathrm{Nm}$. The Runge-Kutta method also fails to preserve the structure of $\left(\mathbb{S}^{2}\right)^{n}$. The mean unit length error is $1.7594 \times 10^{-4}$.

Example 6 (molecular dynamics on a sphere)

We study molecular dynamics on $\mathbb{S}^{2}$. Each molecule is modeled as a particle moving on $\mathbb{S}^{2}$. Molecules are subject to two distinct forces: an attractive force at long range and a repulsive force at short range. Let $m_{i} \in \mathbb{R}$ and $q_{i} \in \mathbb{S}^{2}$ be the mass and the position vector of the $i$ th molecule, respectively. The $i, j$ th element of the inertia matrix is $M_{i j}=m_{i}$ when $i=j$ and $M_{i j}=0$ otherwise. The Lennard-Jones potential is a simple mathematical model that represents the behavior of molecules [26]

$$
V\left(q_{1}, \ldots, q_{n}\right)=\frac{1}{2} \sum_{\substack{i, j=1 \\ j \neq i}}^{n} 4 \varepsilon\left[\left(\frac{\sigma}{\left\|q_{i}-q_{j}\right\|}\right)^{12}-\left(\frac{\sigma}{\left\|q_{i}-q_{j}\right\|}\right)^{6}\right]
$$

where the first term models repulsion between molecules at short distance according to the Pauli principle and the second term models attraction at long distance generated by the van der Walls 


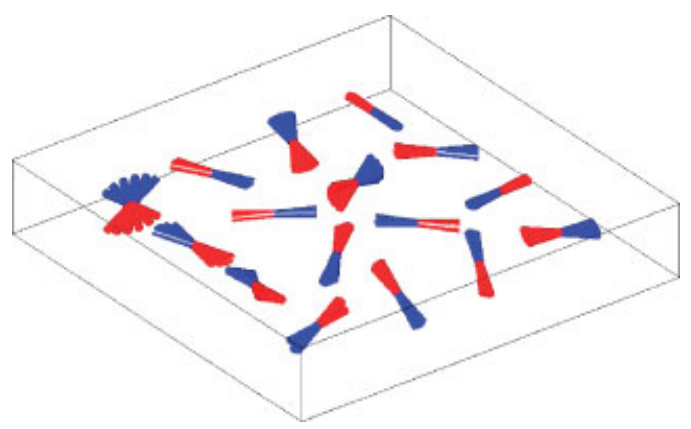

(a)
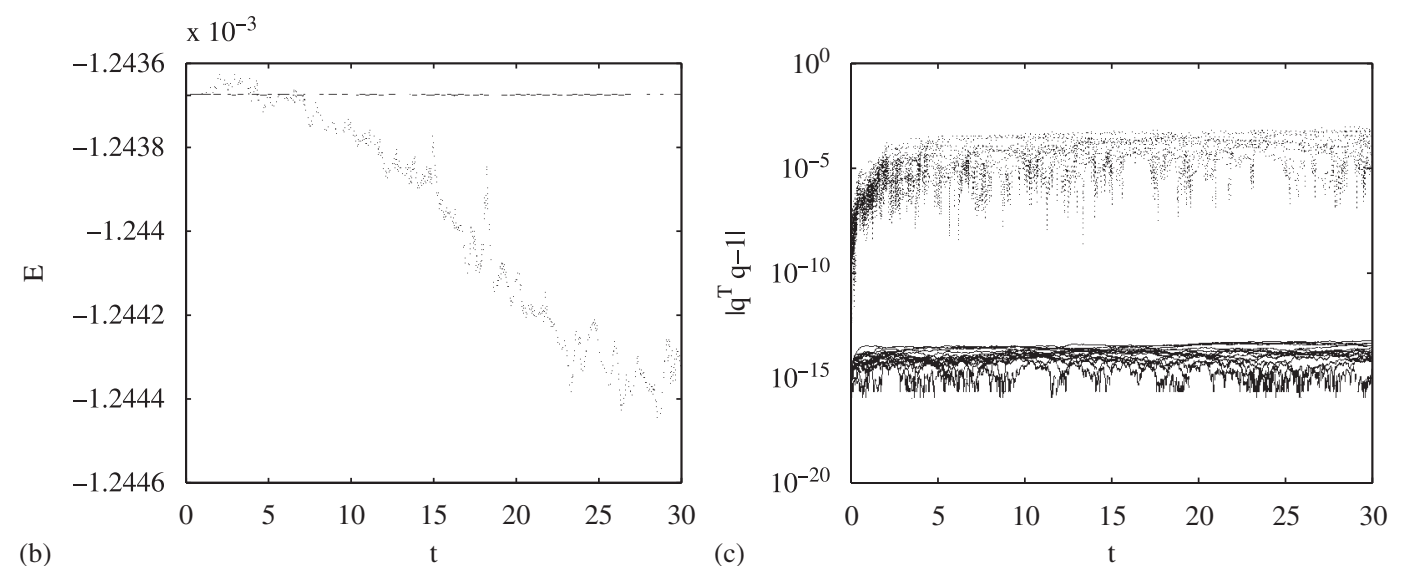

Figure 5. Numerical simulation of an array of magnetic dipoles (RK45: dotted, VI: solid): (a) motion of magnetic dipoles; (b) computed total energy; and (c) unit length error.

forces. The constants $\varepsilon$ and $\sigma$ are molecular constants; $\varepsilon$ is proportional to the strength of the mutual potential and $\sigma$ characterizes inter-molecular force. Substituting these into (7), the continuous equations of motion for the molecular dynamics on a sphere are given by

$$
m_{i} \ddot{q}_{i}=-m_{i}\left(\dot{q}_{i} \cdot \dot{q}_{i}\right) q_{i}-q_{i} \times\left(q_{i} \times \sum_{\substack{j=1 \\ j \neq i}}^{n} 4 \varepsilon \frac{q_{i}-q_{j}}{\left\|q_{i}-q_{j}\right\|}\left[\frac{12 \sigma^{12}}{\left\|q_{i}-q_{j}\right\|^{13}}-\frac{6 \sigma^{6}}{\left\|q_{i}-q_{j}\right\|^{7}}\right]\right)
$$

for $i \in\{1, \ldots, n\}$.

We choose 642 molecules, $n=642$, and we assume each molecule has the same mass, $m_{i}=1$. Initially, molecules are uniformly distributed on a sphere. The strength of the potential is chosen as $\varepsilon=0.01$ and the constant $\sigma$ is chosen such that the inter-molecular force between neighboring molecules is close to zero. The initial velocities are modeled as two vortices separated by $30^{\circ}$. The simulation time is $5 \mathrm{~s}$ and the step size is $h=0.005$.

Trajectories of molecules and the computed total energy are shown at Figure 6. The mean deviation of the total energy is $1.8893 \times 10^{-3}$ and the mean unit length error is $5.2623 \times 10^{-15}$. In molecular dynamics simulations, macroscopic quantities such as temperature and pressure are more useful than the trajectories of molecules. Figure 7 shows the change of kinetic energy distributions 


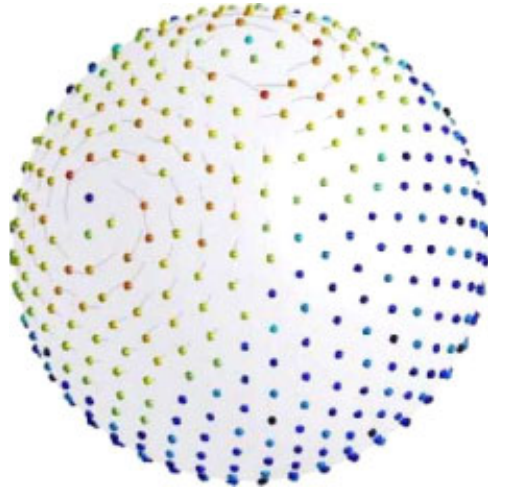

(a)

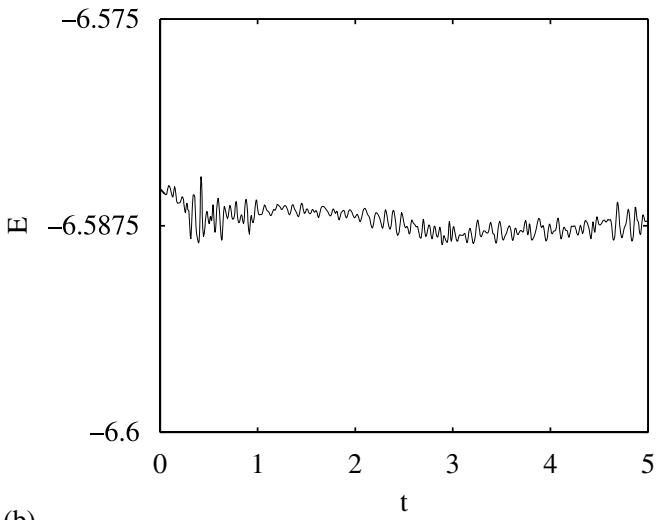

(b)

Figure 6. Numerical simulation of molecular dynamics on a sphere: (a) initial trajectories and (b) computed total energy.

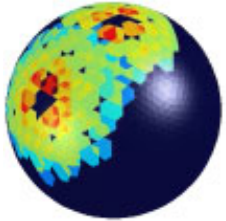

(a)

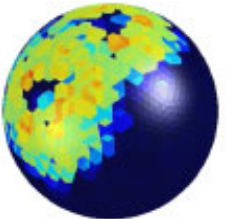

(b)

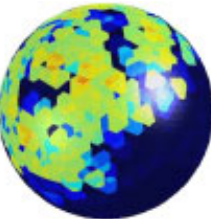

(c)

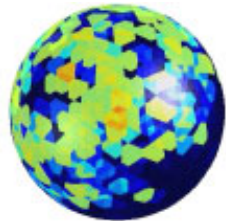

(d)

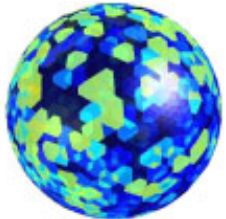

(e)

Figure 7. Kinetic energy distributions over time: (a) $t=0$; (b) $t=0.25$;

(c) $t=0.5$; (d) $t=0.75$; and (e) $t=5$.

over time, which measures the temperature [27]; the sphere is discretized by an icosahedron with 5120 triangular faces and the color of a face is determined by the average kinetic energy of molecules that lie within the face and within its neighboring faces. The local kinetic energy is represented by color shading.

\section{GENERAL THEORY OF LAGRANGIAN MECHANICS ON HOMOGENEOUS SPACES}

In this section, we will describe briefly how the approach adopted in this paper generalizes to arbitrary homogeneous spaces in both continuous and discrete time.

\subsection{Continuous time formulation}

Let $G$ be a Lie group that acts transitively on the homogeneous space $Q$, with an Ad-invariant isotropy subgroup $H_{q}=\{h \in G \mid h \cdot q=q\}$. This induces an isotropy subalgebra $\mathfrak{h} \subset \mathfrak{g}$. We then choose a principle bundle connection $\mathscr{A}: T G \rightarrow \mathfrak{h}$.

Given a Lagrangian system on the homogeneous space described by a Lagrangian $L: T Q \rightarrow$ $\mathbb{R}$ and a distinguished point $q_{0} \in Q$, we consider the horizontal lift of the second-order curve 
$q:[0, T] \rightarrow Q$, with respect to the initial point $q_{0}$, which we denote by $g:[0, T] \rightarrow G$. This is defined by the following system of equations:

$$
q(\cdot)=g(\cdot) q_{0}, \quad \dot{q}(\cdot)=\dot{g}(\cdot) q_{0}, \quad \mathscr{A}(g, \dot{g})=0, \quad g(0)=e
$$

We construct the lifted Lagrangian which, by an abuse of notation, is denoted by $L: T G \rightarrow \mathbb{R}$ and is defined by

$$
L(g, \dot{g})=L\left(g q_{0}, \dot{g} q_{0}\right)
$$

Then, given a solution of Hamilton's principle $(q, \dot{q})$, with initial point $q_{0}$, the horizontal lift of the solution satisfies the following constrained variational principle:

$$
\delta \int L(g, \dot{g}) \mathrm{d} t=0
$$

subject to the constraint that $\mathscr{A}(g, \dot{g})=0$ and $g(0)=e$. The equivalence to Hamilton's principle follows by the construction of the lifted Lagrangian and the horizontal lift. It is important to note that while the lifted curve $g(\cdot)$ on the Lie group depends on the choice of connection, it induces a curve on the homogeneous space through the relation $q(\cdot)=g(\cdot) q_{0}$ that is independent of the choice of connection.

\subsection{Discrete time formulation}

The essential issue in the extension of the prior construction to discrete time is discretizing the constraint induced by the connection. It is possible to formally introduce a discrete connection [28], $\mathscr{A}_{\mathrm{d}}: G \times G \rightarrow H_{g_{1}}$, and impose the discrete horizontal curve condition that is given by $\mathscr{A}_{\mathrm{d}}\left(g_{k}, g_{k+1}\right)=e$.

Then, the discrete horizontal lift of a discrete curve $\left\{q_{k}\right\}_{k=0}^{N}$ is given by $\left\{g_{k}\right\}_{k=0}^{N}$, where

$$
q_{k}=g_{k} q_{0}, \quad \mathscr{A}_{\mathrm{d}}\left(g_{k}, g_{k+1}\right)=e, \quad g_{0}=e
$$

Given a discrete Lagrangian system described by a discrete Lagrangian $L_{\mathrm{d}}: Q \times Q \rightarrow \mathbb{R}$, we construct the lifted discrete Lagrangian $L_{\mathrm{d}}: G \times G \rightarrow \mathbb{R}$ by

$$
L_{\mathrm{d}}\left(g_{k}, g_{k+1}\right)=L_{\mathrm{d}}\left(g_{k} q_{0}, g_{k+1} q_{0}\right)
$$

Then, given the solution of the discrete Hamilton's principle, $\left\{q_{k}\right\}_{k=0}^{N}$, the horizontal lift of the discrete solution satisfies the following constrained discrete variational principle:

$$
\delta \sum_{k=0}^{N-1} L_{\mathrm{d}}\left(g_{k}, g_{k+1}\right)=0
$$

subject to the constraint that $\mathscr{A}_{\mathrm{d}}\left(g_{k}, g_{k+1}\right)=e$ and $g_{0}=e$.

In practice, one can obtain an appropriate discretization of the constraint by introducing a retraction [17], $\varphi_{g}: T_{g} G \rightarrow G$, which satisfies $\varphi_{g}(v)=g$ iff $v=0 \in T_{g} G$ and $\left.\varphi_{g}^{\prime}\right|_{0}=\mathbf{1}_{T_{g} G}$, and then requiring that

$$
\mathscr{A}\left(\varphi_{g_{k}}^{-1}\left(g_{k+1}\right)\right)=0
$$


This is equivalent to the condition in terms of a discrete connection constructed by

$$
\mathscr{A}_{\mathrm{d}}\left(g_{k}, g_{k+1}\right)=\varphi_{g_{k}} \circ \mathscr{A} \circ \varphi_{g_{k}}^{-1}\left(g_{k+1}\right)
$$

As with the continuous time case, while the lifted constrained variational principle on $G$ yields a discrete curve $\left\{g_{k}\right\}_{k=0}^{N}$ that depends on the choice of the discrete connection, it induces a discrete curve on $Q$, through the relation $q_{k}=g_{k} q_{0}$ that is independent of the choice of connection. This is in contrast to integration algorithms for homogeneous spaces that are based on the traditional Lie group methods, wherein the numerical properties of the algorithm depend critically on the choice of connection.

\section{CONCLUSIONS}

Euler-Lagrange equations and variational integrators are developed for Lagrangian mechanical systems evolving on $\left(\mathbb{S}^{2}\right)^{n}$ where the Lagrangian is written in a particular form given by (2). The structure of $\mathbb{S}^{2}$ is carefully considered to obtain global equations of motion on $\left(\mathbb{S}^{2}\right)^{n}$ without local parameterizations or explicit constraints.

In the continuous time setting, this provides a remarkably compact form of the equations of motion compared with the popular angular description. For example, it is not practical to study a triple spherical pendulum by using angles due to the complexity of the trigonometric expressions involved. The global Euler-Lagrange equations on $\left(\mathbb{S}^{2}\right)^{n}$ maintain the same compact structure for arbitrary $n$. In addition to being useful for the theoretical study of global dynamical properties, it is also possible to use them as a finite element model for a continuum problem as shown in Example 4. More generally, combining Lie group or homogeneous variational integrators with noncommutative harmonic analysis techniques will yield multisymplectic variational integrators for problems where either the base space or the fiber is a Lie group or homogeneous space.

The variational integrators on $\left(\mathbb{S}^{2}\right)^{n}$ preserve the geometric properties of the dynamics as well as the structure of the configuration manifold concurrently. They are symplectic, momentum preserving, and they exhibit good energy behavior for exponentially long time as they are derived from discrete Hamilton's principle. Using the characteristics of the homogeneous manifold $\left(\mathbb{S}^{2}\right)^{n}$, the discrete update map is represented by a group action of $\mathrm{SO}(3)$ to obtain compactly represented and, possibly explicit, numerical integrators. In particular, variational integrators on $\left(\mathbb{S}^{2}\right)^{n}$ completely avoid the singularities and complexity introduced by the local parameterizations and explicit constraints.

While the numerical method presented in the paper is equivalent to the RATTLE algorithm with the Lagrange multipliers explicitly eliminated, the methodology proposed is quite general, and can be generalized to higher order. This is achieved by constructing a higher-order Lie group variational integrator [29] through the choice of a higher-order interpolant at the level of the Lie algebra, coupled with a higher-order numerical quadrature formula. The construction presented in this paper allows one to apply the higher-order Lie group integrator to problems on homogeneous spaces. In particular, this will lead to a direct construction for higher-order variational integrators based on an index-reduced formulation of Lagrangian flows on $\left(\mathbb{S}^{2}\right)^{n}$.

At a more abstract level, the procedure described in this paper corresponds to considering a variational principle on a homogeneous space and lifting this to a variational principle at the level of the Lie group. Owing to the isotropy subgroup associated with the action of the Lie group on the homogeneous space, the lift of a curve on the homogeneous space to the Lie group is not 
unique. This can be addressed by introducing a connection that specifies a horizontal space that is complementary to the tangent space of the isotropy subgroup, and requires that the lifted curve is horizontal with respect to the connection.

This means that the lifted variational principle at the level of the Lie group is a constrained variational principle that uses a connection to specify the admissible set of variations. While the solution of the constrained variational principle depends on the choice of connection, we obtain the solution curve on the homogeneous space by the action of the solution curve on the Lie group on the initial point on the homogeneous space and the homogeneous space curve is independent of the choice of connection.

While the construction of variational integrators on $\mathbb{S}^{2}$ in this paper is performed explicitly, the general methodology of lifting a variational principle on a homogeneous space to a constrained variational principle on the Lie group is quite general, and serves as the basis for extending the techniques of Lie group variational integrators to the setting of homogeneous spaces. Furthermore, the general construction is naturally related to continuous and discrete reduction and particularly reduction by stages.

\section{ACKNOWLEDGEMENTS}

We gratefully acknowledge the helpful comments and suggestions of the referees. This paper was supported by the National Science Foundation under grant numbers DMS-0714223, DMS-0726263, DMS-0747659, ECS-0244977, and CMS-0555797.

\section{REFERENCES}

1. Bendersky S, Sandler B. Investigation of a spatial double pendulum: an engineering approach. Discrete Dynamics in Nature and Society 2006; 2006:1-22. DOI: 10.1155/DDNS/2006/25193.

2. Marsden J, Scheurle E, Wendlandt J. Visualization of orbits and pattern evocation for the double spherical pendulum. International Congress on Industrial and Applied Mathematics, Hamburg, Germany, vol. 87, 1995.

3. Hairer E, Lubich C, Wanner G. Geometric Numerical Integration. Springer: Berlin, 2000.

4. McLachlan R, Quispel R. Six lectures on the geometric integration of ODEs. Foundations of Computational Mathematics. London Mathematical Society Lecture Note, vol. 284. Cambridge University Press: Cambridge, 2001; 155-210.

5. Moser J, Veselov A. Discrete versions of some classical integrable systems and factorization of matrix polynomials. Communications in Mathematical Physics 1991; 139:217-243.

6. Marsden J, West M. Discrete mechanics and variational integrators. Acta Numerica, vol. 10. Cambridge University Press: Cambridge, 2001; 317-514.

7. Munthe-Kaas H, Zanna A. Numerical integration of differential equations on homogeneous manifolds. Foundations of Computational Mathematics. Springer: Berlin, 1997; 305-315.

8. Lewis D, Olver P. Geometric integration algorithms on homogeneous manifolds. Foundations of Computational Mathematics 2001; 2(4):363-392. DOI: 10.1007/s102080010028.

9. Lewis D, Nigam N. Geometric integration on spheres and some interesting applications. Journal of Computational and Applied Mathematics 2003; 151(1):141-170. DOI: 10.1016/S0377-0427(02)00743-4.

10. Iserles A, Munthe-Kaas H, Nørsett S, Zanna A. Lie-group methods. Acta Numerica, vol. 9. Cambridge University Press: Cambridge, 2000; 215-365.

11. Shuster M. Survey of attitude representations. Journal of the Astronautical Sciences 1993; 41:439-517.

12. Lee T, Leok M, McClamroch N. Lie group variational integrators for the full body problem in orbital mechanics. Celestial Mechanics and Dynamical Astronomy 2007; 98(2):121-144. DOI: 10.1007/s10569-007-9073-x.

13. Lee T, Leok M, McClamroch N. Lie group variational integrators for the full body problem. Computer Methods in Applied Mechanics and Engineering 2007; 196:2907-2924. DOI: 10.1016/j.cma.2007.01.017.

14. Chikuse Y. State space models on special manifolds. Multivariate Analysis 2006; 97:1284-1294. 
15. Lin D, Yan S, Tang X. Pursuing informative projection on grassmann manifold. Proceedings of the IEEE Computer Society Conference on Computer Vision and Pattern Recognition, New York, NY, 2006; $1727-1734$.

16. Sanz-Serna J. Symplectic integrators for hamiltonian problems: an overview. Acta Numerica 1992; 1:243-286.

17. Shub M. Some remarks on dynamical systems and numerical analysis. Dynamical Systems and Partial Differential Equations (Caracas, 1984). University of Simon Bolivar: Caracas, 1986; 69-91.

18. Kelley C. Iterative Methods for Linear and Nonlinear Equations. SIAM: Philadelphia, PA, 1995.

19. Hairer E. Backward analysis of numerical integrators and symplectic methods. Annals of Numerical Mathematics 1994; 1(1-4):107-132. Scientific computation and differential equations (Auckland, 1993).

20. Andersen H. RATTLE: a 'velocity' version of the SHAKE algorithm for molecular dynamics calculations. Journal of Computational Physics 1983; 52:24-34. DOI: 10.1016/0021-9991(83)90014-1.

21. Yoshida H. Construction of high order symplectic integrators. Physics Letters A 1990; 150:262-268.

22. Wendlandt J, Marsden J. Mechanical integrators derived from a discrete variational principle. Physica D 1997; 106(3-4): 223-246.

23. Kozlov V, Harin A. Kepler's problem in constant curvature spaces. Celestial Mechanics and Dynamical Astronomy 1992; 54:393-399.

24. Hairer E, Lubich C, Wanner G. Geometric numerical integration illustrated by the Störmer-Verlet method. Acta Numerica 2003; 12:399-450.

25. Cheng X, Jalil M, Lee H. Time-quantified monte carlo algorithm for interacting spin array micromagnetic dynamics. Physical Review B 2006; 73:224-438.

26. Lennard-Jones J. Cohesion. The Proceedings of the Physical Society 1931; 43:461-482. DOI: 10.1088/0959$5309 / 43 / 5 / 301$.

27. Allen M, Tildesley D. Computer Simulation of Liquids. Clarendon Press: Oxford, 1987.

28. Leok M, Marsden J, Weinstein A. A discrete theory of connections on principal bundles. Preprint, ArXiv:math.DG/0508338, 2005.

29. Leok M. Generalized Galerkin variational integrators: Lie group, multiscale, and pseudospectral methods. Preprint, ArXiv:math.NA/0508360, 2004. 